TRANSACTIONS OF THE

AMERICAN MATHEMATICAL SOCIETY

Volume 257, Number 1, January 1980

\title{
$K$-THEORY OF HYPERPLANES
}

BY

BARRY H. DAYTON AND CHARLES A. WEIBEL ${ }^{1}$

\begin{abstract}
Let $R$ be the coordinate ring of a union of $N$ hyperplanes in general position in $\mathbf{A}_{k}^{n+1}$. Then

$$
K_{i}(R)=K_{i}(k) \oplus\left(\begin{array}{c}
N-1 \\
n+1
\end{array}\right) K_{n+i}(k) .
$$

This formula holds for $K_{0}, K_{1}, K_{i}(i<0)$, and for the Karoubi-Villamayor groups $K V_{i}(i \in Z)$. For $K_{2}$ there is an extra summand $\bar{R} / R$, where $\bar{R}$ is the normalization of $R$. For $K_{3}$ the above is a quotient of $K_{3}(R)$.

In 84 we show that $K_{1}$-regularity implies $K_{0}$-regularity, answering a question of Bass. We also show that $K_{i}$-regularity is equivalent to Laurent $K_{i}$-regularity for $i<1$. The results of this section are independent of the rest of the paper.
\end{abstract}

This paper is primarily concerned with the computation of the $K$-theory of $N$ hyperplanes in general position in $A^{n+1}$. Our main computation uses the structural similarities to $\mathrm{CW}$ complexes and special configurations which we have suggestively called polysimplicial spheres. To effect the computation we prove some results about $K_{i}$-regularity in $\S 4$. These are independent of the rest of the paper and may be of general interest to $K$-theorists. Finally, we are able to perform a computation of $K_{2}$ using the modified Tate map [9] and 'pointy brackets' $\langle a, b\rangle$. We also show that in this case

$$
N K_{3}(R) \rightarrow K_{3}(R) \rightarrow K V_{3}(R) \rightarrow 0
$$

is exact, giving us a lower bound for $K_{3}$.

Our focus is the following affine scheme. Let $k$ be a field and let $W \subset A^{n+1}$ be the union of hyperplanes $\{V(f) \mid f \in \mathscr{F}\}$. We admit $W$ (or $\mathscr{F}$ ) if it satisfies the following general position conditions: if $V\left(f_{0}\right), \ldots, V\left(f_{p}\right)$ intersect, their normal vectors are linearly independent and we can find $f_{p+1}, \ldots, f_{n} \in \mathcal{F}$ so that the $V\left(f_{i}\right)$ intersect (in a point). The first condition avoids the difficulties encountered in [17]; the second condition avoids 'cylinders' in $W$ and is satisfied if $W$ contains the coordinate hyperplanes. In the case $n=1$, any union of lines in the plane is admissible unless all lines are parallel or three lines meet in a point [5].

In $\$ 1$ we introduce the basic definitions and constructions. Among these is the bookkeeping poset $C$ of all nonempty subschemes of the form

Received by the editors March 20, 1978.

AMS (MOS) subject classifications (1970). Primary 18F25; Secondary 13D15, 16A54.

$K e y$ words and phrases. Karoubi-Villamayor $K$-theory, $K_{i}$-regular, scheme, geometric realization, poset, Tate map.

${ }^{1}$ Second author supported by NSF grant MCS77-18723.

(c) 1980 American Mathematical Society 0002-9947/80/0000-0006/\$06.75 
$V\left(f_{0}, \ldots, f_{p}\right)$. We think of $W$ as being built up from these using the glueing instructions of $\mathcal{C}$, and show that $W$ is the scheme-theoretic colimit of $\mathcal{C}$. In $\$ 2$ we introduce the special case of polysimplicial spheres. These behave toward $K$-theory as spheres do to cohomology. The main technical result is the Extension Theorem 2.4, which allows us to get 'enough' maps of polysimplicial spheres into $W$ to explain the $K$-theory.

$\$ 3$ introduces a genus-like number $g$, counting how many spheres are 'enough'. In the case $k=\mathbf{R}$ we show that the real points $W(\mathbf{R})$ are homotopy equivalent to a $g$-fold wedge of $S^{n}$ 's, so that $g$ counts the number of bounded cells in $W(\mathbf{R})$. In general $|\mathcal{C}| \simeq S^{n} \vee \cdots \vee S^{n}$ ( $g$ copies). If $W$ is in true general position (no parallel hyperplanes) then $g=\left(\begin{array}{c}N-1 \\ n+1\end{array}\right)$. We emphasize the topological analogy because it seems vital to an understanding of the $K$-theory. In analogy with the topological $K$-theory for $\mathrm{CW}$ complexes it seems reasonable that there be a spectral sequence

$$
E_{2}^{p q}=H^{p}\left(|\mathcal{C}| ; K_{-q}(k)\right) \Rightarrow K V_{-p-q}(W)
$$

for reducible varieties $W$ built up by glueing affine pieces together according to the instructions of the poset $\mathcal{C}$. In our case such a spectral sequence would degenerate, giving the correct computations.

$\$ 4$ is an aside on $K_{i}$-regularity, and may be of independent interest to $K$-theorists. We extend a result of Vorst [20] to show that a ring is $F$-regular iff it is Laurent $F$-regular, for $F$ one of $K_{0}, K_{1}, K_{i}(i<0)$, Pic, or $S K_{0}$. This corrects an example of Bass [2]. As a consequence $K_{1}$-regularity implies $K_{0}$-regularity, answering a question of Bass [2]. (Vorst and Van der Kallen have shown this independently [21].) We show that the coordinate ring of $W$ is $K_{1}$-regular, so that the above remarks apply to our situation.

The main computation is performed in $\S 5$. We first show that $K V_{i}(W)=$ $K_{i}(k) \oplus g K_{n+i}(k)$ for $i \in \mathbf{Z}$. This may be written $K V_{*}(W)=K_{*}(k) \otimes$ $H^{-*}(|\mathcal{C}|)$. This is a graded ring isomorphism. Since $K V_{i}(W)=K_{i}(W)$ for $i<1$, this gives us formulas for $K_{0}$ and $K_{1}$ as well as $K_{-n}(W)=\mathbf{Z}^{8}$, $K_{i}(W)=0$ for $i<-n$.

The last section computes $K_{2}(W)$. Our primary tool here is the modified Tate map from $K_{2}(W)$ to $\Omega_{W / k}^{2}$. Using a computation of Dennis-Krusemeyer [7] we identify generators for the kernel of $K_{2}(W) \rightarrow K V_{2}(W)$ and find relations using the Tate map. Finally, we use a spectral sequence argument to obtain the information on $K_{3}(W)$ mentioned above.

Since the arguments are not complicated by weakening the hypothesis on $k$, we have done so. Until $\S 4, k$ is any commutative ring with unit and no nilpotent elements. For $\S 5$, it is $K_{1}$-regular, while for $\S 6$, it is $K_{1}$ - and $K_{2}$-regular.

1. Algebra of hyperplanes. Throughout this section, $k$ denotes a commutative ring with unit. Given $A=k\left[x_{0}, \ldots, x_{n}\right]$, we consider the set $\operatorname{LIN}(A)$ of polynomials of degree one in $A$. Note that $\operatorname{LIN}(A)$ is invariant under linear changes of variables. Let $X=\left\{x_{0}, \ldots, x_{n}\right\}$. We call a subset $Y$ of $\operatorname{LIN}(A)$ a 
coordinate system if there is a bijection $\nu: X \rightarrow Y$, inducing a $k$-algebra automorphism of $A$ by $x \mapsto \nu(x)$.

DefinITION 1.1. A finite nonempty subset $\mathscr{F}$ of $\operatorname{LIN}(A)$ is called admissible if every $\sigma \subseteq \mathcal{F}$ either generates $A$ or is a subset of a coordinate system contained in $\mathscr{F}$. We say that $\mathscr{F}$ is in general position if each $\sigma \subseteq \mathcal{F}$ with $n+1$ elements is a coordinate system.

Here are some examples of admissible sets. $X$ is admissible. The 'cube' $\{x, x-1 \mid x \in X\}$ and the ' $n$-simplex' $X \cup\left\{x_{0}+\cdots+x_{n}-1\right\}$ are admissible. If $n=0$, a finite nonempty set $\left\{x-b_{i}\right\}$ is admissible iff each $b_{i}-b_{j}$ is a unit. The 'admissible sets' of [5] are equivalent to our admissible sets when $n=1$.

The following facts about admissible sets will be useful. We first note that every admissible set $\mathscr{F}$ contains a coordinate system, so we can apply a linear automorphism to $A$ to assume $X \subseteq \mathcal{F}$. In fact, if $\sigma \subset \mathcal{F}$ has $p$ elements and $\sigma A \neq A$ we can assume $\sigma=\left\{x_{0}, \ldots, x_{p-1}\right\}$ so that $A / \sigma A \cong k\left[x_{p}, \ldots, x_{n}\right]$.

Proposition 1.2. Let $\mathcal{F}$ be an admissible set containing $X$ and $f=\Sigma a_{i} x_{i}+$ $b$. If $b=0, f \in X$. Otherwise $b$ is $a$ unit, and the $a_{i}$ are either units or nilpotent.

Proof. Assume $f \notin X$. Then $X$ and $f$ are comaximal, so $b$ must be a unit of $k$. It is now enough to show that $a_{0}$ is either a unit or 0 nilpotent. Consider $\sigma=\{f\} \cup X-\left\{x_{0}\right\}$, and note that $A / \sigma A=k\left[x_{0}\right] /\left(a_{0} x_{0}+b\right)$ must be either 0 or $k$. This is true only when $a_{0}$ is a unit or nilpotent. Done.

Remark. Of course when $k$ is a field, Proposition 1.2 is vacuous. See [5] for further conditions on the coefficients when $n=1$. One would have similar conditions when $n>1$. To avoid notational complications we will assume $k$ has no nilpotent elements.

Proposition 1.3. If $\mathscr{F}$ is admissible, every subset $\mathcal{G}$ of $\mathscr{F}$ containing a coordinate system is admissible.

Proof. Let $Y$ be a coordinate system, $Y \subseteq \mathcal{G} \subseteq \mathcal{F}$. Let $\sigma \subseteq \mathcal{G}$ be maximal with respect to $\sigma A \neq A$; we have to show that $\sigma$ is a coordinate system. Assume otherwise and pick coordinates so that $\sigma \subset X \subseteq \mathcal{F}$, and $x_{0} \notin \sigma$. Write

$$
y_{i}=\sum_{j} a_{i j} x_{j}+b_{i} \quad(i=0, \ldots, n) .
$$

As $Y$ is a coordinate system, $\operatorname{det}\left(a_{i j}\right)$ is a unit of $k$. In particular, some $a_{i 0}$ must be a unit by Proposition 1.2. This yields a contradiction to the maximality of $\sigma: \sigma \subseteq X-\left\{x_{0}\right\}$ so $y_{i} \notin \sigma A$ yet

$$
\sigma A+y_{i} A \subseteq\left(a_{i 0} x_{0}-b_{i}, x_{1}, \ldots, x_{n}\right) \neq A .
$$

Hence maximal elements of $\mathcal{G}$ re $\sigma A \neq A$ are coordinate systems, i.e., $\mathcal{G}$ is admissible.

Suppose that $f_{0} \in \operatorname{LIN}(A)$, so that $A / f_{0} A$ is a polynomial algebra over $k$. If $\left\{f_{0}, \ldots, f_{n}\right\}$ is a coordinate system for $A$ in $\operatorname{LIN}(A)$ and $\rho: A \rightarrow A / f_{0} A$, then 
$\left\{\rho\left(f_{1}\right), \ldots, \rho\left(f_{n}\right)\right\}$ is a coordinate system for $A / f_{0} A$. We define $\operatorname{LIN}\left(A / f_{0} A\right)$ relative to such a coordinate system. Note that $\operatorname{LIN}\left(A / f_{0} A\right)$ is independent of the choice of coordinate system; it is $\rho(\operatorname{LIN}(A))-k$.

If $\mathscr{F} \subset \operatorname{LIN}(A)$, we let $\mathscr{F} \mid f_{0}=\rho(\mathscr{F}) \cap \operatorname{LIN}\left(A / f_{0} A\right)$. Passing from $\mathscr{F}$ to $\mathscr{F} \mid f_{0}$ corresponds to intersecting the hyperplanes of $\mathscr{F}-\left\{f_{0}\right\}$ with the affine space $V\left(f_{0}\right)$. When $\mathscr{F}$ is admissible, for each $g \in \mathcal{F} \mid f_{0}$ there is a unique $f \in \mathcal{F}$ for which $\rho(f)=g$, and there is a well-defined set map $\rho^{-1}: \mathscr{F} \mid f_{0} \rightarrow \mathscr{F}$.

Proposition 1.4. Let $\mathscr{F} \subset \operatorname{LIN}(A)$ be admissible, $n \geqslant 1$. Then $\mathscr{F} \mid f_{0}$ is admissible for each $f_{0} \in \mathscr{F}$.

Proof. We have remarked that $A / f_{0} A$ is a polynomial algebra and $\mathscr{F} \mid f_{0}$ is a finite subset of $\operatorname{LIN}\left(A / f_{0} A\right)$. Let $f_{1}, \ldots, f_{p} \in \mathscr{F}$ be such that $\rho\left(f_{1}\right), \ldots, \rho\left(f_{p}\right) \in \mathscr{F} \mid f_{0}$, and set $\sigma=\left\{f_{1}, \ldots, f_{p}\right\}$. If $\rho(\sigma)$ does not generate $A / f_{0} A, \sigma A+f_{0} A \neq A$. By the admissibility of $\mathscr{F}$ there are $f_{p+1}, \ldots, f_{n} \in \mathscr{F}$ so that the $f$ 's form a coordinate system for $A$. By the above remarks, $\rho(\sigma)$ is a subset of the coordinate system $\rho\left(f_{1}\right), \ldots, \rho\left(f_{n}\right)$ for $A / f_{0} A$ in $\mathscr{F} \mid f_{0}$. Done.

Similarly, if $\sigma \subset \operatorname{LIN}(A)$ is a proper subset of a coordinate system, we can make sense of $\operatorname{LIN}(A / \sigma A)$. If $\mathscr{F} \subset \operatorname{LIN}(A)$, we write $\mathscr{F} \mid \sigma$ for $\rho(\mathscr{F}) \cap$ $\operatorname{LIN}(A / \sigma A)$. Since $\mathscr{F}\left|\sigma=\left(\left(\left(\mathscr{F} \mid f_{0}\right) \mid f_{1}\right) \ldots\right)\right| f_{p}$ when $\sigma=\left\{f_{0}, \ldots, f_{p}\right\}$ we see by the proposition that if $\mathscr{F}$ is admissible, so is $\mathscr{F} \mid \sigma$ whenever $\sigma \subset \mathscr{F}, A \neq \sigma A$ and $\operatorname{card}(\sigma)<n+1$.

Another useful construction for creating admissible sets is the orthogonal sum $\mathscr{F} \# \mathcal{G}$. If $\mathscr{F}$ is admissible for $A, \mathcal{G}$ admissible for $B$, then $\mathscr{F} \# \mathcal{G}$ is the set of $f \otimes 1, f \in \mathcal{F}$, and $1 \otimes g, g \in \mathcal{G}$, in $\operatorname{LIN}\left(A \otimes_{k} B\right)$. If $\sigma \subset \mathcal{F}, \tau \subset \mathcal{G}$ are coordinate systems for $A$ and $B$, then $\sigma \otimes 1 \cup 1 \otimes \tau$ is a coordinate system for $A \otimes_{k} B$. From this it follows that $\mathscr{F} \sharp \mathcal{G}$ is admissible.

The rest of this section will deal with partially ordered sets (posets) associated with admissible sets. If $\mathscr{F} \subseteq \operatorname{LIN}(A)$, we let $\mathcal{C}(\mathscr{F})$ denote the poset of nonempty finite subsets $\sigma$ of $\mathscr{F}$ for which $\sigma A \neq A$. We have already noted that $\mathscr{F}$ is admissible if and only if $\mathscr{F}$ is finite and maximal elements of $\mathcal{C}(\mathscr{F})$ are coordinate systems for $A$.

We shall call a finite nonempty subposet $\mathbb{Q}$ of $\mathcal{C}(\operatorname{LIN}(A))$ an $A$-poset. In addition to $\mathcal{C}(\mathscr{F})$ we shall be interested in the following two $A$-posets. If $\operatorname{card}(\mathscr{F}) \neq 1$, then $\mathcal{C}(\mathscr{F})-\left\{f_{0}\right\}$ is an $A$-poset. If $f_{0} \in \mathscr{F}$ we will identify (by abuse of notation) the comma category $f_{0} \downarrow \mathcal{C}(\mathscr{F})$ with the $A$-poset of all $\sigma \in \mathcal{O}(\mathcal{F})$ for which $f_{0} \in \sigma$.

If $Q$ is an $A$-poset, $\mathscr{B}$ a $B$-poset then we can define the $A \otimes B$-poset $\mathbb{Q} \# \mathscr{B}$ as all $\sigma \otimes 1,1 \otimes \tau$, and $\sigma \otimes 1 \cup 1 \otimes \tau$ with $\sigma \in \mathbb{Q}, \tau \in \mathscr{B}$. Note that we have the formula $\mathcal{C}(\mathscr{F}) \# \mathcal{C}(\mathcal{G})=\mathcal{C}(\mathscr{F} \# \mathcal{G})$.

Let $\mathcal{Q}$ be an $A$-poset, $\mathscr{B}$ a $B$-poset, where $A, B$ are polynomial $k$-algebras. A $\mathscr{P}$-morphism from $\mathcal{Q}$ to $\mathscr{B}$ is a pair $(\lambda, \varphi)$, where $\lambda: B \rightarrow A$ is a $k$-algebra morphism and $\varphi: \mathbb{Q} \rightarrow \mathscr{B}$ is a poset map, subject to the following condition. For each $\sigma \in \mathbb{Q}$ the set $\lambda \varphi(\sigma)$ must lie in the ideal $\sigma A$.

When $\mathbb{Q}=\mathcal{C}(\mathscr{F}), \mathscr{B}=\mathcal{C}(\mathcal{G})$ we can define a poset map $\varphi: \mathbb{Q} \rightarrow \mathscr{B}$ by a map $\mathscr{F} \rightarrow \mathcal{G}$ which by abuse we shall also call $\varphi$. The formula reads: 
$\varphi(\sigma)=\{\varphi(f) \mid f \in \sigma\}$. In this case $(\lambda, \varphi)$ is a $\mathscr{P}$-morphism iff $f$ divides $\lambda \varphi(f)$ for each $f \in \mathscr{F}$.

There is a category $\mathscr{P}$ of posets and $\mathscr{P}$-morphisms. The objects are all $A$-posets as $A$ ranges over the polynomial algebras of $k$. We shall think of objects of $\mathcal{P}$ as giving glueing data for a union of affine spaces in the following way.

Given an $A$-poset $\mathscr{Q}$ we define a functor $R=R(\mathscr{Q})$ from $\mathbb{Q}$ to $k$-algebras by $R(\sigma)=A / \sigma A$, letting $R(\sigma \subset \tau)$ be the canonical projection from $A / \sigma A$ to $A / \tau A$. We define $k[\mathscr{Q}]=\lim R(\mathscr{Q})$.

Given a morphism $(\lambda, \varphi): \mathbb{Q} \rightarrow \mathscr{B}$ in $\mathscr{P}$, the morphisms $\lambda(\sigma): B / \varphi(\sigma) B \rightarrow$ $A / \sigma A$ induced by $\lambda$ give a natural transformation from $R(\mathscr{B}) \varphi$ to $R(\mathscr{Q})$, and hence a $k$-algebra homomorphism $(\lambda, \varphi)^{*}$ from $k[\mathscr{B}]$ to $k[\mathscr{Q}]$. From this we easily verify the

LEMMA 1.5. There is a contravariant functor $k[\cdot]$ from $\mathscr{P}$ to $k$-algebras and a covariant functor $\operatorname{Spec}(k[\cdot])$ from $\mathscr{P}$ to (affine) $k$-schemes.

1.6. Here are several examples of $\mathscr{P}$-morphisms we shall find useful.

(1) When $A=B$ and $\mathscr{Q}$ is a subposet of $\mathscr{B}$, there is a $\mathscr{P}$-morphism $(1, \subseteq)$ : $\mathcal{Q} \rightarrow \mathscr{B}$. In particular, if $\mathbb{Q}$ is the discrete poset of minimal elements of $\mathscr{B}=\mathcal{C}(\mathscr{F})$, where $\mathscr{F}=\left\{f_{1}, \ldots, f_{p}\right\}$, then $k[\mathscr{Q}]=A / f_{1} A \oplus \cdots \oplus A / f_{p} A$ and $k[\Re] \rightarrow k[\mathscr{Q}]$ is an injection. We shall see later that (if $k$ is integrally closed) $k[\mathbb{Q}]$ is the integral closure of $k[\mathfrak{B}]$.

(2) When $f_{0} \in \mathscr{F}, \mathscr{F}$ admissible for $A$, then there is a $\mathscr{P}$-morphism $(\rho, \theta)$ from $\mathcal{C}\left(\mathscr{F} \mid f_{0}\right)$ to $\mathcal{C}(\mathscr{F})$ given by $\rho: A \rightarrow A / f_{0} A$ and $\theta(\sigma)=\left\{f_{0}\right\} \cup \rho^{-1}(\sigma)$, where $\rho^{-1}: \mathscr{F} \mid f_{0} \rightarrow \mathscr{F}$. We shall see in Theorem 1.11 that Spec $k[\mathcal{C}(\mathscr{F})]$ is the union of the hyperplanes $V(f), f \in \mathscr{F}$, in the affine space $\operatorname{Spec}(A)$. The map $\operatorname{Spec}(\rho, \theta)^{*}$ identifies $\operatorname{Spec} k\left[\mathcal{C}\left(\mathscr{F} \mid f_{0}\right)\right]$ with the union of the subschemes $V\left(f_{0}, f\right)$ of $V\left(f_{0}\right), f \in \mathscr{F}-\left\{f_{0}\right\}$.
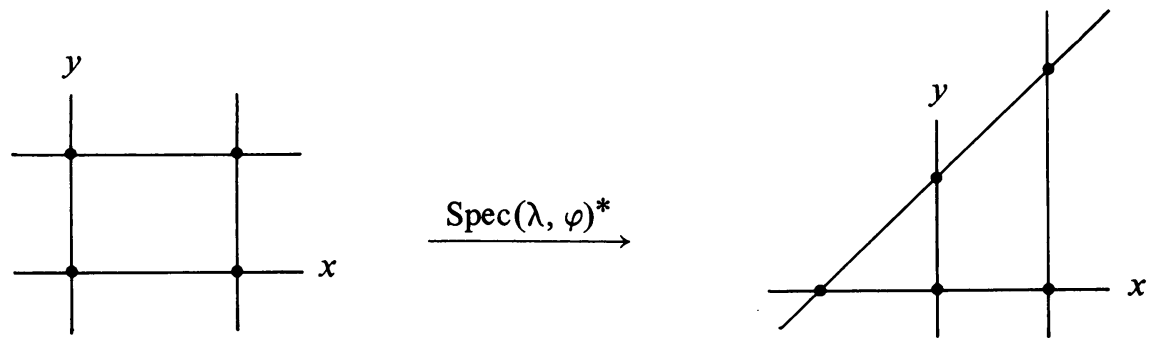

Figure 1

(3) The following example shows that neither $\lambda$ nor $(\lambda, \varphi)^{*}$ need be a surjection. This map was described on p. 18 of [16]. Let $A=k[x, y]$, $\operatorname{char}(k)$ $\neq 2, \mathbb{Q}=\mathcal{C}(\mathscr{F})$ and $\mathcal{C}=\mathcal{C}(\mathcal{G})$, where $\mathscr{F}=\{x, y, x-1, y-1\}, \mathcal{G}=\{x, y$, $x-1, x-y+1\}$. Let $\lambda: A \rightarrow A$ be given by $\lambda(x)=x, \lambda(y)=(x+1) y$, and let $\varphi$ be induced by the map $\mathscr{F} \rightarrow \mathcal{G}$ fixing $x, y, x-1$ and sending $y-1$ to $x-y+1$. Then $(\lambda, \varphi): \mathbb{Q} \rightarrow \mathcal{C}$ is a $\mathscr{P}$-morphism, and is illustrated in 
Figure 1. In Theorem 1.11 we shall see that $k[\mathcal{Q}]=A / F A, k[\mathcal{C}]=A / G A$, where $F=x y(x-1)(y-1)$ and $G=x y(x-1)(x-y+1)$. It is now a simple matter to check that neither $\lambda$ nor $(\lambda, \varphi)^{*}$ is onto. We remark in passing that $(\lambda, \varphi)$ factors through the subposet $\mathscr{B}=\mathcal{C}-\{y, x-y+1\}$ of $\mathcal{C}$, inducing an isomorphism $k[\mathscr{B}] \rightarrow k[\mathscr{Q}]$ if $\frac{1}{2} \in k$.

Proposition 1.7. Let $\mathbb{Q}, \mathscr{B}$ be $A$-posets satisfying: if $\sigma \subset \tau$ in $\mathbb{Q} \cup \mathscr{B}$ then $\sigma \in \mathbb{Q}$ (resp. $\mathscr{B}$ ) implies $\tau \in \mathbb{Q}$ (resp. $\mathscr{B}$ ). Then the square (induced by the inclusions)

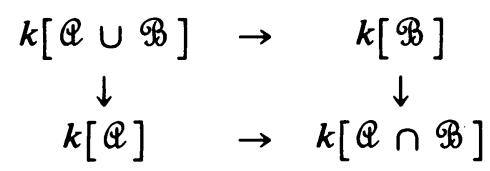

is cartesian. If $\mathbb{Q} \cap \mathscr{B}=\varnothing$ we let the lower right term be 0 .

REMARK. The hypothesis implies that $\mathscr{Q} \cup \mathscr{B}$ is the poset pushout of $\mathscr{Q}$ and $\mathscr{B}$ over their intersection. Hence the proposition may be paraphrased as saying that in this case $k[\cdot]$ converts pushouts to pullbacks.

Proof. Set $\mathcal{C}=\mathbb{Q} \cup \mathscr{B}, \mathscr{D}=\mathbb{Q} \cap \mathscr{B}$. If $\mathscr{D}=\varnothing$ it is clear that $k[\mathcal{C}]=$ $k[\mathscr{Q}] \oplus k[\mathscr{B}]$, and the result follows. Let $C$ be the pullback and define $h(\sigma)$ : $C \rightarrow A / \sigma A$ for each $\sigma \in \mathcal{C}$ as follows. If $\sigma \in \mathbb{Q}$ we use $C \rightarrow k[\mathbb{Q}] \rightarrow A / \sigma A$; if $\sigma \in \mathscr{B}$ we use $C \rightarrow k[\mathscr{B}] \rightarrow A / \sigma A$. If $\sigma \in \mathscr{D}$ both choices agree since they coincide with $C \rightarrow k[\mathscr{D}] \rightarrow A / \sigma A$. The hypothesis implies that if $\sigma \subset \tau$ in $\bigodot$ the map $h(\tau)$ is $h(\sigma)$ followed by $A / \sigma A \rightarrow A / \tau A$. Thus $h: C \rightarrow k[\mathcal{C}]$ is well defined, and it is clear that it is an inverse to the functorial $k[\mathcal{C}] \rightarrow C$. Done.

The following application of Proposition 1.7 will play a crucial role in $\$ 5$. Let $\mathscr{F} \subset \operatorname{LIN}(A)$ be admissible, $n>1$, and $f \in \mathscr{F}$ be such that $\mathscr{F}-\{f\}$ is also admissible. Then $\mathcal{C}=\mathcal{C}(\mathscr{F})$ is the union of $f \downarrow \mathcal{C}$ and $\mathcal{C}-\{f\}$, and these satisfy the condition of (1.7). Moreover, their intersection is the image $(\rho, \theta) \mathcal{C}(\mathcal{F} \mid f)$.

We now compute the result of applying $k[\cdot]$. Now $f \downarrow \mathcal{C}$ has an initial object, so $k[f \downarrow \mathcal{C}]=A / f A$ and the map $(1, \subseteq)^{*}: k[\mathcal{C}] \rightarrow k[f \downarrow \mathcal{C}]=A / f A$ is the natural one. Since the indexing categories are isomorphic under $\theta$ we have $k[(\rho, \theta) \mathcal{C}(\mathscr{F} \mid f)]=k[\mathcal{C}(\mathscr{F} \mid f)]$. Finally, we claim that $k[\cdot]$ of $(1, \subseteq)$ : $\mathcal{C}(\mathscr{F}-\{f\}) \rightarrow \mathcal{C}-\{f\}$ is an isomorphism. Since both categories have the same initial objects, $(1, \subseteq)^{*}$ is an injection. Now $(1, \sigma \mapsto \sigma-\{f\}): \mathcal{C}-\{f\}$ $\rightarrow \mathcal{C}(\mathscr{F}-\{f\})$ is a left inverse for $(1, \subseteq)$ in $\mathscr{P}$, which implies that $(1, \subseteq)^{*}$ is surjective as well.

The proposition now tells us that

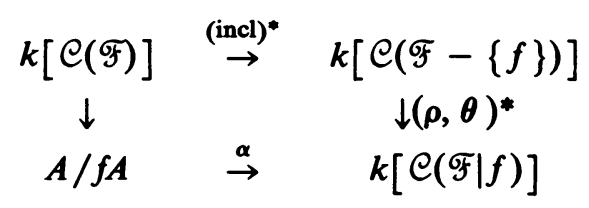

is cartesian, where $\alpha$ is the structural map for $\mathcal{C}(\mathscr{F} \mid f)$. We shall see later 
(Theorem 1.11) that $\alpha$ and the left vertical map, hence all four ring maps, are onto.

We now insert some technical results to expedite our computations.

Proposition 1.8. Let $\mathscr{F} \subset \operatorname{LIN}(A)$ be admissible. Then for any subset $S$ of $\mathscr{F}$ (IIS)A $=\cap\{f A \mid f \in S\}$.

Proof. We proceed by induction on $\operatorname{card}(S)$, the result being trivial for $\operatorname{card}(S)=0$ or 1 . Let $S=\left\{f_{0}, \ldots, f_{p}\right\}, F=f_{1} \cdots f_{p}$, so that $\Pi S=f_{0} F$. Inductively $\cap f_{i} A=f_{0} A \cap F A$, so we have to show that $f_{0} A \cap F A \subseteq f_{0} F A$.

Now the admissibility of $\mathscr{F}$ implies that for each $f \in \mathscr{F}-\left\{f_{0}\right\}$ either $\left(f_{0}, f\right)$ is an $A$-sequence or $f_{0}$ and $f$ are comaximal. In either case, $f$ is a non-zero-divisor modulo $f_{0}$. Hence $F=f_{1} \cdots f_{p}$ is a non-zero-divisor modulo $f_{0}$. Thus if $F h \in f_{0} A$ we must have $h=f_{0} g$, and so $F h \in f_{0} F A$, as desired.

Corollary 1.9. Let $g, f_{1}, \ldots, f_{p} \in \mathcal{F}, \mathcal{F}$ admissible.

$$
\bigcap_{i=1}^{p}\left(f_{i}, g\right) A=\left(f_{1} \cdots f_{p}, g\right) A .
$$

Proof. We consider $\rho: A \rightarrow A / g A$, and show that $\cap \rho\left(f_{i} A\right)$ is generated by $\rho\left(f_{1} \cdots f_{p}\right)$. If any $\rho\left(f_{i}\right)=0$, the result is trivial. If any $\rho\left(f_{i}\right)$ is a unit we can ignore it. This takes care of the case $A=k[g]$. Thus we can assume that the $\rho\left(f_{i}\right)$ belong to $\mathscr{F} \mid g$. By Proposition $1.4, \mathscr{F} \mid g$ is admissible, and the result follows from Proposition 1.8 applied to $A / g A$.

We now restrict ourselves to the following special case. Let $\mathcal{F}=$ $\left\{f_{0}, \ldots, f_{N}\right\}$ be an admissible set for $A$, and let $\mathcal{C}=\mathcal{C}(\mathscr{F})$. Inspection of $\mathcal{C}$ reveals that $k[\mathcal{C}]$ is completely determined by the $A / \sigma A$ for singleton and doubleton $\sigma$. In fact,

$$
k[\mathcal{C}]=\left\{\left(\bar{a}_{0}, \ldots, \bar{a}_{N}\right) \in \prod A / f_{i} A \mid \forall i, j a_{i} \equiv a_{j} \bmod \left(f_{i}, f_{j}\right)\right\} .
$$

Now for each $\sigma \in \mathcal{C}$ there is a natural structural map $A \rightarrow A / \sigma A$; these maps induce a canonical map $\alpha: A \rightarrow k[\mathcal{C}]$. Note that $F=\Pi \mathscr{F}=f_{0} \cdots f_{N}$ lies in $\operatorname{ker}(\alpha)$.

THEOREM 1.11. Let $\mathcal{F}$ be admissible for A. Then $\alpha$ induces an isomorphism $A / F A \simeq k[\mathcal{C}(\mathscr{F})]$.

Proof. From the description of $k[\mathcal{C}]$ in (1.10) and Proposition 1.8, we see that $\operatorname{ker}(\alpha)=F A$. It remains to show that $\alpha$ is a surjection. Let $a_{0}, \ldots, a_{N} \in$ $A$ be such that $\left(\bar{a}_{0}, \ldots, \bar{a}_{N}\right)$ lies in $k[\mathcal{C}]$. We produce inductively $b_{p} \in A$ for which $b_{p} \equiv a_{i} \bmod \left(f_{i}\right), i=0, \ldots, p$. The case $p=0$ is satisfied by $b_{0}=a_{0}$. Given $b=b_{p-1}$, note that $b \equiv a_{p} \bmod \left(f_{i}, f_{p}\right)$ for $i<p$. If we write $F_{p}=$ $f_{0} \cdots f_{p-1}$, Corollary 1.9 yields

$$
a_{p}-b \in \bigcap_{i=0}^{p-1}\left(f_{i}, f_{p}\right)=\left(F_{p}, f_{p}\right) .
$$

Write $a_{p}-b=g F_{p}+h f_{p}$ and set $b_{p}=b+g F_{p}$. This completes the inductive step. We are done, as $\alpha\left(b_{N}\right)=\left(\bar{a}_{0}, \ldots, \bar{a}_{N}\right)$. 
Proposition 1.12. Assume $k$ is integrally closed. Let $\mathscr{F}=\left\{f_{0}, \ldots, f_{N}\right\}$ be admissible for $A$, and let $F_{i}=\Pi \mathscr{F}-\left\{f_{i}\right\}$. Then the normalization of $k[\mathcal{C}(\mathscr{F})]$ is $\Pi A / f_{i} A$, and the conductor is

$$
\left(F_{0}, \ldots, F_{N}\right)=\bigcap_{i=0}^{N}\left(f_{i}, F_{i}\right)=\bigcap_{i, j}\left(f_{i}, f_{j}\right) .
$$

Proof. This follows from [12], given Theorem 1.11, so we only sketch the proof. Now $\alpha\left(F_{i}\right) / \alpha\left(F_{i}+f_{i}\right)=(0, \ldots, 1,0, \ldots)$ lies in the quotient ring of $k[\mathcal{C}]$ and $\Pi A / f_{i} A$ is an integral extension, so it is the normalization.

From (1.10) it is clear that $\cap\left(f_{i}, f_{j}\right)$ is the conductor (we have identified elements of $A$ with their images under $\alpha)$. This equals $\cap\left(f_{i}, F_{i}\right)$ by Corollary 1.9; a componentwise check and Proposition 1.8 yield the remaining identity. Done.

2. Polysimplicial spheres. We now introduce the 'building blocks' for admissible sets.

Definition 2.1. The simplicial $n$-sphere, $\delta(n)$, is the $k\left[x_{0}, \ldots, x_{n}\right]$-poset $\mathcal{C}\left(\left\{x_{0}, \ldots, x_{n}, x_{0}+\cdots+x_{n}-1\right\}\right)$. Given $n_{1}, \ldots, n_{k}>0, k>1$, set $n=$ $k-1+\Sigma n_{i}$. The polysimplicial $n$-sphere $\delta\left(n_{1}, \ldots, n_{k}\right)$ is the orthogonal product $\delta\left(n_{1}\right) \# \ldots \# \delta\left(n_{k}\right)$. Any object of $\mathcal{P}$ isomorphic to $\delta(n)$ or $\delta\left(n_{1}, \ldots, n_{k}\right)$ will be called a (poly-) simplicial $n$-sphere. Thus if we choose coordinates $x_{i j}, i=1, \ldots, k, j=0, \ldots, n_{i}$, for $A=k\left[\left\{x_{i j}\right\}\right], \delta\left(n_{1}, \ldots, n_{k}\right)$ is isomorphic to the $A$-poset $\mathcal{C}(\mathscr{F})$, where $\mathscr{F}=\left\{x_{i j}\right\} \cup\left\{y_{1}, \ldots, y_{k}\right\}, y_{i}=x_{i 0}$ $+\cdots+x_{i n_{i}}-1$. Note that $\mathscr{F}$ is an admissible set with $n+k+1$ elements. If $f \in \mathscr{F}$ we will write $\mathcal{S} \mid f$ for $\mathcal{C}(\mathscr{F} \mid f)$.

REMARK. Let $\Delta^{n+1}$ denote the $n+1$-simplex $x_{i} \geqslant 0, \sum x_{i}<1$ in $\mathbf{R}^{n+1}$. If $k=\mathbf{R}$ the set of real points of $\operatorname{Spec}(\mathbf{R}[\delta(n)])$ is the linear span of $\partial \Delta^{n+1}$, and the set of real points of $\operatorname{Spec}\left(\mathbf{R}\left[\delta\left(n_{1}, \ldots, n_{k}\right)\right]\right)$ is the linear span of $\partial\left(\Delta^{n_{1}+1}\right.$ $\left.\times \cdots \times \Delta^{n_{k}+1}\right)$ in $\mathbf{R}^{n+1}$. Note that $\partial\left(\Delta^{n_{1}+1} \times \cdots \times \Delta^{n_{k}+1}\right)$ is homeomorphic to the $n$-sphere $S^{n}$, whence the name. The polysimplicial $n$-sphere $\delta(0, \ldots, 0)$ represents the (boundary of the) $n$-cube. When $n=2$ there are exactly 3 different polysimplicial spheres: the boundaries of the 3-simplex $(\delta(2))$, 3-cube $(\delta(0,0,0))$, and prism $(\delta(1,0) \simeq \delta(0,1))$.

LEMMA 2.2. If $\mathcal{S}$ is a polysimplicial $n$-sphere, $n \neq 0$, then $\mathcal{S} \mid f$ is a polysimplicial $(n-1)$-sphere for each $\{f\} \in \mathcal{S}$.

Proof. Let $\delta=\mathcal{C}(\mathcal{F})=\delta\left(n_{1}, \ldots, n_{k}\right)$, so that $\delta \mid f$ means $\mathcal{C}(\mathcal{F} \mid f)$. By choosing a new coordinate system we can assume that $f=x_{10}$. If $n_{1}=0$, $\delta \mid x_{10} \simeq \mathcal{S}\left(n_{2}, \ldots, n_{k}\right)$. On the other hand it is easy to verify that $\delta(n) \mid x_{0} \simeq$ $\delta(n-1)$, so if $n_{1}>1$ we have $\delta \mid x_{10} \simeq \delta\left(n_{1}-1, n_{2}, \ldots, n_{k}\right)$.

LEMMA 2.3. If $\mathscr{F}$ is an admissible set in general position, $\operatorname{card}(\mathscr{F})=n+2$, then $C(\mathscr{F}) \simeq \delta(n)$. 
Proof. Choose a coordinate system so that $\mathscr{F}=X \cup\{f\}, f=\Sigma a_{i} x_{i}+b$, $A=k[X]$. By Proposition 1.2 and the general position hypothesis, $b$ and the $a_{i}$ are all units. Define $(\lambda, \varphi): \delta(n) \rightarrow \mathcal{C}(\mathcal{F})$ by $\varphi\left(x_{i}\right)=x_{i}, \varphi\left(\Sigma x_{i}-1\right)=f$, and $\lambda\left(x_{i}\right)=b x_{i} / a_{i}$. Then $\lambda \varphi\left(x_{i}\right)=b x_{i} / a_{i}$ and $\lambda \varphi\left(\sum x_{i}-1\right)=\lambda(f)=$ $b\left(\sum x_{i}-1\right)$, so $(\lambda, \varphi)$ is a $\mathscr{P}$-morphism. A straightforward verification shows that $\left(\lambda^{-1}, \varphi^{-1}\right)$ is $(\lambda, \varphi)^{-1}$.

EXTENSION THEOREM 2.4. Let $\mathscr{F}$ be admissible for $A \simeq k\left[x_{0}, \ldots, x_{n}\right], n \neq 0$. Let $f_{0} \in \mathcal{F}$ be such that some coordinate system of $\mathcal{F}$ does not contain $f_{0}$. Assume that $\delta\left(n_{1}, \ldots, n_{k}\right)$ is a polysimplicial $(n-1)$-sphere and that we are given a $\mathcal{P}$-morphism $\left(\lambda^{\prime}, \varphi^{\prime}\right): \mathcal{S}\left(n_{1}, \ldots, n_{k}\right) \rightarrow \mathcal{C}\left(\mathscr{F} \mid f_{0}\right), \varphi^{\prime}$ injective. Then there is a polysimplicial $n$-sphere $\mathcal{S}$, an $\{s\} \in \mathcal{S}$ for which $\mathcal{S}\left(n_{1}, \ldots, n_{k}\right) \simeq$ $\mathcal{S} \mid s$, and $a \mathcal{P}$-morphism $(\lambda, \varphi): \mathcal{S} \rightarrow \mathcal{C}(\mathscr{F})$ such that the following diagram commutes:

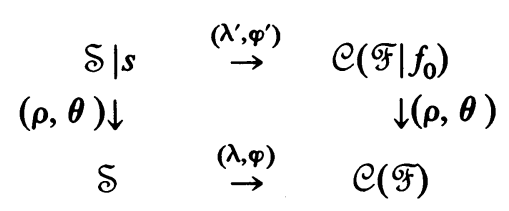

The vertical morphisms are those of 1.6, example (2), and $\varphi$ is injective.

Proof. Set $X=\left\{x_{i j} \mid 0 \leqslant j \leqslant n_{i}, i=1, \ldots, k\right\}$, coordinates for $\delta\left(n_{1}, \ldots, n_{k}\right)$. By dimensionality $\varphi^{\prime}$ maps singletons to singletons. Choose the coordinate system $\left\{f_{0}\right\} \cup \rho^{-1} \varphi^{\prime}(X)$ for $A$ and write $x_{i j}=\rho^{-1} \varphi^{\prime}\left(x_{i j}\right)$ so that $A=k\left[X, f_{0}\right]$. For $i=1, \ldots, k$ set $f_{i}=\rho^{-1} \varphi^{\prime}\left(x_{i 1}+\ldots-1\right) \in \mathscr{F}$ and write $f_{i}=a_{i} f_{0}+g_{i}$, where $a_{i} \in k, g_{i} \in k[X]$.

Case 1 . All the $a_{i}=0$. Now some coordinate system in $\mathscr{F}$ does not contain $f_{0}$ so there exists some $f \in \mathcal{F}-\left\{f_{0}, \ldots, f_{k}\right\}$ of the form $f=a f_{0}-g$, $0 \neq a \in k, g \in k[X]$. Set $s=x_{0}, \delta=\delta\left(0, n_{1}, \ldots, n_{k}\right)$ and let $\iota: k[X] \rightarrow$ $k\left[X, x_{0}\right]$ be the standard inclusion. Define $\varphi\left(x_{0}\right)=f_{0}, \varphi\left(x_{0}-1\right)=f, \varphi\left(x_{i j}\right)=$ $x_{i j}, \varphi\left(y_{i}\right)=f_{i}$ so that $\varphi \theta=\theta \varphi^{\prime}$. Define $\lambda$ by $\lambda\left(f_{0}\right)=\lambda \lambda^{\prime}(g) x_{0} / a, \lambda\left(x_{i j}\right)=$ $\iota \lambda^{\prime} \rho\left(x_{i j}\right)$ so that $\rho \lambda=\lambda^{\prime} \rho$. It is easy to see that $(\lambda, \varphi)$ is a $\mathscr{P}$-morphism: $\lambda \varphi\left(x_{i j}\right)=\iota \lambda^{\prime} \varphi^{\prime}\left(x_{i j}\right), \lambda \varphi\left(y_{i}\right)=\lambda\left(f_{i}\right)=\lambda \lambda^{\prime} \rho\left(g_{i}\right)=u \lambda^{\prime} \varphi^{\prime}\left(y_{i}\right), \lambda \varphi\left(x_{0}\right)=\lambda(g) x_{0} / a$, and $\lambda \varphi\left(x_{0}-1\right)=\lambda(g)\left(x_{0}-1\right)$. This establishes Case 1 .

Case 2. Some $a_{i} \neq 0$. After permuting coordinates we can assume $a_{1} \neq 0$. Set $s=x_{1, n_{1}+1}, \mathcal{S}=\mathcal{S}\left(n_{1}+1, n_{2}, \ldots, n_{k}\right)$, considered as a $k[X, s]$-poset. Define $\varphi: \mathcal{S} \rightarrow \mathcal{C}(\mathcal{F})$ by extending $\varphi(s)=f_{0}, \varphi\left(x_{i j}\right)=x_{i j}, \varphi\left(y_{i}\right)=f_{i}$. As $\theta(\sigma)=\left\{f_{0}\right\} \cup \rho^{-1}(\sigma)$ and $f_{i}=\rho^{-1} \varphi^{\prime}\left(\rho\left(y_{i}\right)\right)$ we have $\theta \varphi^{\prime}=\varphi \theta$.

We define $\lambda$ by giving coherent maps $\lambda(\sigma)$ from $A / \varphi(\sigma) A$ to $R(\sigma)=$ $k[X, s] /(\sigma)$ for each $\sigma \in \mathcal{S}$. This will yield a map $\bar{\lambda}: A \rightarrow k[\varphi(\mathcal{S})] \rightarrow k[\delta]$. By Theorem $1.11, k[X, s] \rightarrow k[\delta]$ is onto, so we can lift to a map $\lambda: A \rightarrow k[X, s]$. The construction makes it clear that $(\lambda, \varphi)$ is a $\mathscr{P}$-morphism. If $\lambda(\{s\})$ is $\lambda^{\prime}$ : $A / f_{0} A \rightarrow k[X]$ then we will have $\rho \lambda=\lambda^{\prime} \rho$, and the displayed diagram will commute. 
We begin by defining $\lambda(\{s\})=\lambda^{\prime}$ and if $\sigma=\theta(\tau)$ we set $\lambda(\sigma)=\lambda^{\prime}(\tau)$ : $A / \varphi(\sigma)=\left(A / f_{0} A\right) /\left(\varphi^{\prime}(\tau)\right) \rightarrow k[X] /(\tau)$. These are well defined and coherent since $\left(\lambda^{\prime}, \varphi^{\prime}\right)$ is a $\mathcal{P}$-morphism. If $\operatorname{card}(\sigma)=n+1$ we must have $\lambda(\sigma)=$ id: $k \rightarrow k$. We proceed to define the $\lambda(\sigma)$ for $\sigma \in \mathcal{S}, s \notin \sigma$ by induction on $n+1-\operatorname{card}(\sigma)$. Fix $\sigma$.

Inductively, whenever $\sigma \subset \tau$ in $\mathcal{S}$ the map $\lambda(\tau)$ is defined coherently. Now $\delta \mid \sigma$ is an admissible $R(\sigma)$-poset, and the $\lambda(\tau)$ induce a map $A / \varphi(\sigma) A \rightarrow$ $k[\mathcal{C}(\mathscr{F}) \mid \varphi(\sigma)] \rightarrow k[\delta \mid \sigma]$, which we lift to $\lambda(\sigma): A / \varphi(\sigma) A \rightarrow R(\sigma)$ using Theorem 1.11. The construction makes it clear that if $\sigma \subset \tau$ the diagram

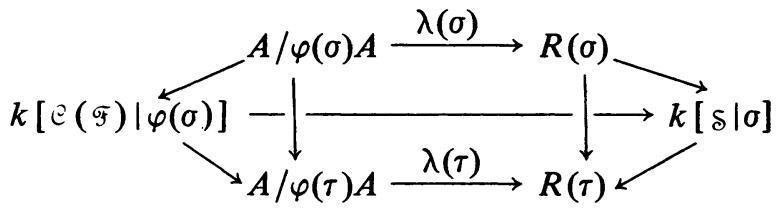

commutes, completing the inductive step. Done.

REMARK. There is another possibility for the choice of $\mathcal{\delta}$. Even if some $a_{i} \neq 0$, it may be the case that some $f \in \mathscr{F}-\left\{f_{0}\right\}$ has the property that, whenever $\rho(\sigma)$ is a coordinate system in $\operatorname{im}\left(\varphi^{\prime}\right), \sigma \cup\{f\}$ is a coordinate system in $\mathscr{F}$. This was true of the $f$ in Case 1 , since $A / \sigma A=k[f]$ for such a $\sigma$. In this case we could have set $\delta=\delta\left(0, n_{1}, \ldots, n_{k}\right), \varphi(s)=f_{0}, \varphi(s-1)=f$, $\varphi\left(x_{i j}\right)=x_{i j}, \varphi\left(y_{i}\right)=f_{i}$ and defined $\lambda$ by bootstrapping. Since $\varphi$ is a poset isomorphism, the construction of $\lambda$ is exactly as in Case 2. Example 1.6(3) illustrates this case.

Corollary 2.5. Let $\mathscr{F}=\left\{f_{i j} \mid i=1, \ldots, k, 0<j<n_{i}+1\right\}$ be an admissible set for which $\sigma \in \mathcal{F}$ lies in $\mathcal{C}(\mathcal{F})$ whenever some $f_{i j} \notin \sigma$ for each $i$. Then there is a $\mathscr{P}$-morphism $(\lambda, \varphi): \mathcal{S}\left(n_{1}, \ldots, n_{k}\right) \rightarrow \mathcal{C}(\mathscr{F})$ with $\varphi\left(x_{i j}\right)=f_{i j}, \varphi\left(y_{i}\right)=$ $f_{i, n_{i}+1}$.

Proof. Changing coordinates we can assume $f_{i j}=x_{i j}, j \neq n_{i}+1$, and let $X=\left\{x_{i j} \mid(i, j) \neq(1,0)\right\}$. Write $\varphi\left(y_{1}\right)=a x_{10}-b+g$, where $b, a \in k$ and $g \in k[X]$ has no constant term. Note $a \neq 0, b \neq 0$ because $\left\{\varphi\left(y_{1}\right)\right\} \cup X$ is a coordinate system, and $\left\{x_{10}, \varphi\left(y_{1}\right)\right\} \cup X$ is comaximal. Define $\left(\lambda^{\prime \prime}, \varphi^{\prime \prime}\right)$ : $\delta(0) \rightarrow \mathcal{C}(\mathcal{F})$ by $\varphi^{\prime \prime}(x)=x_{10}, \varphi^{\prime \prime}(x-1)=\varphi^{\prime \prime}\left(y_{1}\right), \lambda^{\prime \prime}(X)=0, \lambda^{\prime \prime}\left(x_{10}\right)=$ $b x / a$. This is a $\mathcal{P}$-morphism. We now apply the above remark, feeding in the $f_{i 0}$ and $\varphi\left(y_{i}\right)$, to get a $\mathcal{P}$-morphism $\left(\lambda^{\prime}, \varphi^{\prime}\right): \mathcal{S}(0, \ldots, 0) \rightarrow \mathcal{C}(\mathscr{F})$. Finally, we feed in the remaining $f_{i j}$ and apply Case 2 of the Extension Theorem.

Definition 2.6. Let $\mathscr{F}$ be admissible for $A \simeq k\left[x_{0}\right], N=\operatorname{card}(\mathscr{F})$. A basic set of 0 -spheres for $\mathscr{F}$ is a set $\Sigma$ of $N-1$ morphisms $\left(\lambda_{i}, \varphi_{i}\right): \delta(0) \rightarrow \mathcal{C}(\mathcal{F})$ with the property that for some indexing $f_{1}, \ldots, f_{N}$ of $\mathscr{F}$ and some indexing $i=2, \ldots, N$ for $\Sigma$ we have $\left\{\varphi_{i}(x), \varphi_{i}(x-1)\right\}=\left\{f_{i}, f_{j}\right\}$ with $j<i$ for each $i$.

Inductively, a basic set of $n$-spheres for an admissible set $\mathscr{F}$ of $A \simeq$ $k\left[x_{0}, \ldots, x_{n}\right]$ is a set $\Sigma$ of morphisms $\left(\lambda_{i}, \varphi_{i}\right): \mathcal{S}_{i} \rightarrow \mathcal{C}(\mathscr{F})$, the $\mathcal{S}_{i}$ being polysimplicial $n$-spheres, with the following properties. If $\operatorname{card}(\mathscr{F})=n+1, \Sigma$ 
is the empty set. Otherwise, there is an $f \in \mathscr{F}$ for which $\mathscr{F}-\{f\}$ is admissible and $\Sigma-\Sigma_{0}$ is a basic set of $n$-spheres for $\mathscr{F}-\{f\}$. Here

$$
\Sigma_{0}=\left\{\left(\lambda_{i}, \varphi_{i}\right) \in \Sigma \mid \varphi\left(\left\{s_{i}\right\}\right)=\left\{f_{i}\right\} \text { for some } s_{i}\right\} .
$$

Moreover, we require the set of all $\left(\lambda_{i}, \varphi_{i}\right)\left|s_{i}: \delta_{i}\right| s_{i} \rightarrow \mathcal{C}(\mathcal{F} \mid f),\left(\lambda_{i}, \varphi_{i}\right) \in \Sigma_{0}$, to be a basic set of $(n-1)$-spheres for $\mathscr{F} \mid f$.

This requires that each $\varphi_{i}$ be a poset injection, so that $s_{i}$ is well defined by $\varphi_{i}\left(s_{i}\right)=f$. The morphism $\left(\lambda_{i}, \varphi_{i}\right) \mid s_{i}$ is defined by the induced map $\lambda_{i}^{\prime}: A / f A \rightarrow$ $k[X] /\left(s_{i}\right)$ and by $\varphi_{i}^{\prime}(\tau)=\theta_{i}^{-1} \varphi_{i} \theta(\tau)$. This makes sense since $\theta_{i}: \mathcal{S}_{i} \mid s_{i} \rightarrow \mathcal{S}_{i}$ is an injection onto all $\sigma \in \mathcal{S}_{i}$ containing $s_{i}$. We shall see in $\S 3$ that a basic set of spheres has a nice geometric interpretation, and in $\$ 5$ that it has a nice $K$-theoretic interpretation as well.

Proposition 2.7. If $\mathcal{F}$ is admissible, a basic set of $n$-spheres for $\mathcal{F}$ exists.

Proof. If $\operatorname{card}(\mathscr{F})=n+1$ the empty set is basic. If $n=0$ we use Corollary 2.5 to get $\left(\lambda_{i}, \varphi_{i}\right): \delta(0) \rightarrow \mathcal{C}\left(\left\{f_{1}, f_{i}\right\}\right) \subseteq \mathcal{C}(\mathscr{F})$ for $i=$ $2, \ldots, \operatorname{card}(\mathcal{F})$. Otherwise we can pick $f \in \mathscr{F}$ so that $\mathscr{F}-\{f\}$ and $\mathscr{F} \mid f$ are admissible, using Propositions 1.3, 1.4. Inductively, we can find basic sets $\Sigma_{1}$ for $\mathscr{F}-\{f\}, \Sigma_{2}$ for $\mathscr{F} \mid f$. By the Extension Theorem, we can extend $\Sigma_{\mathbf{2}}$ to a set $\Sigma_{0}$ of morphisms $\mathcal{S}_{i} \rightarrow \mathcal{C}(\mathscr{F})$. Then $\Sigma=\Sigma_{1} \cup \Sigma_{0}$ is basic for $\mathscr{F}$.

3. Geometry of hyperplanes. In this section we introduce the invariant $g(\mathscr{Q})$ for $A$-posets. Restricting to the case $Q=C(F)$ for $\mathscr{F}$ admissible, we show that the geometric realization $|\mathscr{Q}|$ of the nerve of the category $\mathbb{Q}$ is homotopy equivalent to a $g(\mathbb{Q})$-fold wedge of $n$-spheres. When $n=1$, the space $|\mathbb{Q}|$ is exactly the graph $G$ used in [15]. We also show that when $k=\mathbf{R}, g(\mathscr{Q})$ counts the number of bounded cells in $\mathbf{R}^{n+1}$ of the real points of $\operatorname{Spec}(\mathbf{R}[\mathbb{Q}]) . \mathbf{L}$. Roberts has remarked upon this relationship [14], [16] for $n<2$.

The topological results of this section are to be considered as analogues of the $K$-theory. Since the poset $\mathcal{C}(\mathscr{F})$ is a bookkeeping device, it is not surprising that its cohomology should be connected with the $K$-theory.

Definition 3.1. Let $A \simeq k\left[x_{0}, \ldots, x_{n}\right]$, and let $\mathbb{Q}$ be an $A$-poset. For each $\sigma \in \mathbb{Q}$ we set $i(\sigma)=n+1-\operatorname{card}(\sigma)$, and $i(\varnothing)=n+1$. For $i=0, \ldots, n$ let $m_{i}$ denote the number of $\sigma \in \mathbb{Q}$ with $i=i(\sigma)$, i.e., $A / \sigma A \simeq k\left[x_{1}, \ldots, x_{i}\right]$. Define

$$
g(\mathbb{Q})=(-1)^{n+1}+\sum_{i=0}^{n}(-1)^{i} m_{i}=\sum(-1)^{i(\sigma)}
$$

where the latter summation is over all $\sigma \in \mathbb{Q} \cup\{\varnothing\}$.

The standard pigeonhole argument yields

Lemma 3.2. Fix $A$ and let $\mathcal{Q}, \mathscr{B}$ be A-posets. Then $g(\mathscr{Q} \cup \mathscr{B})+g(\mathscr{Q} \cap \mathscr{B})$ $=g(\mathbb{Q})+g(\mathscr{B})$. Here we take $g(\mathbb{Q} \cap \mathscr{B})=(-1)^{n+1}$ if $\mathbb{Q} \cap \mathscr{B}=\varnothing$.

LEMMA 3.3. $g\left(\mathbb{Q}^{\prime} \# \mathbb{Q}^{\prime \prime}\right)=g\left(\mathbb{Q}^{\prime}\right) g\left(\mathbb{Q}^{\prime \prime}\right)$. 
Proof. Let $\mathbb{Q}^{\prime}$ be an $A^{\prime}$-poset, $\mathbb{Q}^{\prime \prime}$ an $A^{\prime \prime}$-poset, and set $\mathbb{Q}=\mathbb{Q}^{\prime} \# \mathbb{Q}^{\prime \prime}$, $A=A^{\prime} \otimes A^{\prime \prime}$. The elements of $\mathbb{Q} \cup\{\varnothing\}$ are all unions $\sigma \cup \tau, \sigma \in \mathbb{Q}^{\prime} \cup$ $\{\varnothing\}, \tau \in \mathbb{Q}^{\prime \prime} \cup\{\varnothing\}$. As $A /(\sigma \cup \tau) A=A^{\prime} / \sigma A^{\prime} \otimes A^{\prime \prime} / \tau A^{\prime \prime}$ we have $i(\sigma \cup \tau)$ $=i^{\prime}(\sigma)+i^{\prime \prime}(\tau)$. Writing $d(t)=\Sigma\left\{t^{i(\sigma)}, \sigma \in \mathbb{Q} \cup\{\varnothing\}\right\}$, it follows that $d(t)=$ $d^{\prime}(t) d^{\prime \prime}(t)$, where $d^{\prime}, d^{\prime \prime}$ are defined similarly. The lemma now results from setting $t=-1$.

LEMMA 3.4. $g(\mathcal{C}(X))=0$.

Proof. Here

$$
m_{i}=\left(\begin{array}{c}
n+1 \\
i
\end{array}\right)
$$

so

$$
g=\sum\left(\begin{array}{c}
n+1 \\
i
\end{array}\right)(-1)^{i}=(1-1)^{n+1}=0 .
$$

Proposition 3.5. Let $\mathscr{F}$ be an admissible set in general position. If card(F) $=N$ then

$$
g(\mathcal{C}(\mathscr{F}))=\left(\begin{array}{c}
N-1 \\
n+1
\end{array}\right)
$$

Proof. We induct on $N-n>1$, the case $N=n+1$ being Lemma 3.4. Set $g(n, N)=g(\mathcal{C}(\mathscr{F}))$. From

$$
m_{i}=\left(\begin{array}{c}
N \\
n+1-i
\end{array}\right)
$$

we obtain

$$
\begin{aligned}
g(n, N) & =\left(\begin{array}{c}
N \\
n+1
\end{array}\right)-\left(\begin{array}{l}
N \\
n
\end{array}\right)+\cdots \pm\left(\begin{array}{c}
N \\
1
\end{array}\right) \mp\left(\begin{array}{c}
N \\
0
\end{array}\right) \\
& =\left(\begin{array}{c}
N \\
n+2
\end{array}\right)-g(n+1, N) .
\end{aligned}
$$

Note that $g(n, N)$ does not depend on $\mathscr{F}$. Inductively we know $g(n+1, N)$ and compute

$$
g(n, N)=\left(\begin{array}{c}
N \\
n+2
\end{array}\right)-\left(\begin{array}{c}
N-1 \\
n+2
\end{array}\right)=\left(\begin{array}{c}
N-1 \\
n+1
\end{array}\right) .
$$

COROLlaRY 3.6. If $\mathcal{S}$ is a polysimplicial sphere, $g(\delta)=1$.

Proof. By Lemma 3.3 it is enough to do the case $\mathcal{S}=\mathcal{S}(n)$. By Lemma 2.3, the proposition applies with $N=n+2$ to give $g(\delta(n))=1$.

Proposition 3.7. Let $n \neq 0, f \in \mathscr{F}$ be such that $\mathscr{F}$ and $\mathscr{F}-\{f\}$ are admissible. Then $g(\mathcal{C}(\mathcal{F}))=g(\mathcal{C}(\mathcal{F}-\{f\}))+g(\mathcal{C}(\mathscr{F} \mid f))$.

Proof. We again formally include $\varnothing$ in all posets. Then $\mathcal{C}(\mathscr{F})$ is the disjoint union (on objects) of $\mathcal{C}(\mathscr{F}-\{f\}) \cup\{\varnothing\}$ and $\theta(\mathcal{C}(\mathscr{F} \mid f) \cup\{\varnothing\})$. If $f \notin \sigma, i(\sigma)$ is the same for $\mathscr{F}$ and $\mathscr{F}-\{f\}$, while for $\sigma \in \mathcal{C}(\mathscr{F} \mid f) \cup\{\varnothing\}$ we have $i(\sigma)=i(\theta(\sigma))$. The result follows from $g=\Sigma(-1)^{i(\sigma)}$.

Corollary 3.8. If $\mathscr{F}$ is admissible, $g(\mathcal{C}(\mathscr{F}))>0$. 
Proof. We induct on $N=\operatorname{card}(\mathscr{F})$ and $n$. When $n=0, g=m_{0}-1>0$, and when $N=n+1$ we invoke Lemma 3.4. If $N>n+1$ there is some $f \in \mathscr{F}$ not in all coordinate systems of $\mathscr{F}$, and by Proposition $1.3, \mathscr{F}-\{f\}$ is admissible. The result now follows by Proposition 3.7 and our inductive assumption.

We now consider the realization $|\mathcal{C}(\mathcal{F})|$ of the nerve of the category $\mathcal{C}(\mathscr{F})$. For elementary properties of this functor, we refer the reader to pp. 81-84 of [13]. We shall need the following simple topological fact.

LEMMA 3.9. Let $S$ be a subcomplex of a contractible $\mathrm{CW}$ complex $C$. If $S \rightarrow X$ is a null-homotopic map to some space $X$, then $X \cup_{S} C \simeq X \vee \Sigma S$, where $\Sigma$ denotes suspension.

THEOREM 3.10. Let $\mathscr{F}$ be an admissible set for $A \cong k\left[x_{0}, \ldots, x_{n}\right]$. Then $|\mathcal{C}(\mathscr{F})|$ is homotopy equivalent to a wedge of $g=g(\mathcal{C}(\mathscr{F}))$ copies of $S^{n}$.

Proof. We use induction on $n$ and $N=\operatorname{card}(\mathscr{F})$. For $n=0,|\mathcal{C}(\mathscr{F})|$ is $N$ discrete points and $g=N-1$. When $N=n+1$ the category $\mathcal{C}(\mathscr{F})$ has terminal object $\mathscr{F}$, so that $|\mathcal{C}(\mathscr{F})|$ is contractible, while $g=0$ by Lemma 3.4. We can thus assume $n \neq 0, X \varsubsetneqq \mathscr{F}$ and pick $f \in \mathscr{F}-X$. By (1.3), $\mathscr{F}-\{f\}$ is admissible, so inductively $|\mathcal{C}(\mathscr{F}-\{f\})|$ is h.e. to a wedge of $g_{1}=$ $g(\mathcal{C}(\mathscr{F}-\{f\}))$ copies of $S^{n}$. Also by induction $|\mathcal{C}(\mathscr{F} \mid f)|$ is h.e. to a $g_{2}$-fold wedge of $(n-1)$-spheres, $g_{2}=g(\mathcal{C}(\mathscr{F} \mid f))$.

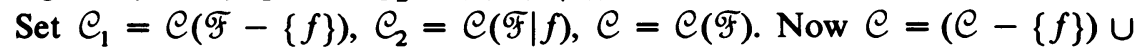

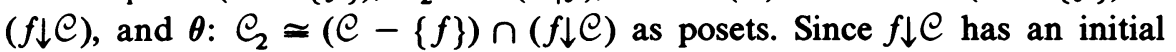
object $|f \downarrow \mathcal{C}|$ is contractible. Moreover, the natural transformation $\sigma \mapsto \sigma-$ $\{f\}$ on $\mathcal{C}-\{f\}$ induces a deformation retraction of $|\mathcal{C}-\{f\}|$ to $\left|\mathcal{C}_{1}\right|$. In particular, the inclusion $|\theta|:\left|\bigodot_{2}\right| \rightarrow|\mathcal{C}-\{f\}|$ is null homotopic. Induction, Lemma 3.9, and Proposition 3.7 now combine to show that $|\mathcal{C}|$ is a wedge of $g_{1}+g_{2}=g(\mathcal{C})$ copies of $S^{n}$.

COROLlaRY 3.11. $|\mathcal{S}| \simeq S^{n}$ for a polysimplicial $n$-sphere $\mathfrak{\delta}$.

THEOREM 3.12. Let $\mathcal{F}$ be an admissible set for $A=\mathbf{R}\left[x_{0}, \ldots, x_{n}\right]$, and let $W(\mathscr{F}) \subset \mathbf{R}^{n+1}$ be the union of the hyperplanes $V(f)$ given by $\mathscr{F}$. Then $W(\mathscr{F})$ is h.e. to a wedge of $g(\mathcal{C}(\mathcal{F})) n$-spheres.

Proof. We again use induction on $n$ and $N$. For $n=0, W(\mathscr{F})$ is $N$ discrete points. For $N=n+1$, linear contraction to the unique vertex shows $W(\mathscr{F})$ is contractible. We can thus assume $n \neq 0$, and that $\mathscr{F}-\{f\}$ is admissible for some $f \in \mathscr{F}$.

Now $W(\mathcal{F})=W(\mathcal{F}-\{f\}) \cup V(f), V(f)$ is contractible, and $V(f) \cap$ $W(\mathscr{F}-\{f\}) \cong W(\mathscr{F} \mid f)$. Invocation of Lemma 3.9, induction, and Proposition 3.7 finishes the proof.

We can be more precise about the nature of the homotopy equivalence in Theorem 3.10, using Corollary 3.11 and the existence of a basic set of polysimplicial spheres for $\mathscr{F}$. 
Proposition 3.13. Let $\Sigma$ be a basic set of spheres for $\mathscr{F}$. Then $\Sigma$ has exactly $g(\mathcal{C}(\mathscr{F}))$ elements $\left(\lambda_{i}, \varphi_{i}\right): \mathcal{S}_{i} \rightarrow \mathcal{C}(\mathscr{F})$. These induce a homotopy equivalence

$$
\bigvee S^{n} \simeq \bigvee\left|\delta_{i}\right| \sim|\mathcal{P}(\mathscr{F})|
$$

In particular, they induce the reduced cohomology isomorphism

$$
\tilde{H}^{*}(|\mathcal{C}(\mathscr{F})|) \rightarrow \bigoplus \tilde{H}^{*}\left(\left|\delta_{i}\right|\right) \simeq g \tilde{H}^{*}\left(S^{n}\right) .
$$

Proof. We follow the proof of Theorem 3.10 using the data $f, \Sigma_{0}$ supplied by $\Sigma$. Inductively, we have $\operatorname{card}(\Sigma)=\operatorname{card}\left(\Sigma_{0}\right)+\operatorname{card}\left(\Sigma-\Sigma_{0}\right)=g(\mathcal{C}(\mathscr{F} \mid f))$ $+g(\mathcal{C}(\mathcal{F}-\{f\}))$, which is $g(\mathcal{C}(\mathscr{F}))$ by Proposition 3.7. Moreover, the maps for $\Sigma-\Sigma_{0}$ and $\Sigma_{0} \mid f$ induce homotopy equivalences. Note that (unless $n=0$, when the proof is trivial) the space $|\mathcal{C}(\mathscr{F})|$ is path-connected so any map $\mathrm{II}\left|\delta_{i}\right| \rightarrow|\mathcal{C}|$ factors through $\mathrm{V}\left|\delta_{i}\right|$ up to homotopy. The data for $\Sigma$ show that each map from $\left|\left(\mathscr{S}_{i} \mid s_{i}\right)\right|$ extends to a map from its extension $\left|\delta_{i}\right|$ into $|\mathcal{C}(\mathscr{F})|$. From the proof of Theorem 3.10 we see that these new maps realize the remaining $g_{2}$-fold wedge of $S^{n}$ 's.

REMARK. When $k=\mathbf{R}$, the same proof shows that $\bigvee S^{n} \simeq \bigvee W\left(\delta_{i}\right) \rightarrow$ $W(\mathscr{F})$ is also a homotopy equivalence.

4. $K_{i}$-regularity. In this section we answer question (III) of [2], correcting an example of Bass in the process. We also develop the tools necessary for the main computation in $\$ 5$.

$K_{i}$ and $K V_{i},-\infty<i<\infty$, refer to the Bass-Milnor-Quillen [1], [13] and Karoubi-Villamayor [11] $K$-theories, respectively. Recall from [2] that a ring $R$ is $K_{i}$-regular if $K_{i}(R)=K_{i}\left(R\left[t_{1}, \ldots, t_{n}\right]\right)$ for all $n>0$ and Laurent $K_{i}$-regular if $R\left[t_{1}, t_{1}^{-1}, \ldots, t_{n}, t_{n}^{-1}\right]$ is $K_{i}$-regular for all $n>0$. The motivation is that any regular ring is $K_{i}$-regular for $-\infty<i<\infty$. Note that $R\left[t_{1}, \ldots, t_{n}\right]$ is $K_{i}$-regular if $R$ is.

The following is a consequence of "Vorst's Theorem" (Theorem 1.1 of [20]) and contracted functors [1, XII, \$7].

THEOREM 4.1. Let $R$ be an associative ring, $-\infty<i<1$.

(a) If $N K_{i}(R)=0, N K_{i}\left(R_{S}\right)=0$ for every central multiplicatively closed set (m.c.s.) $S$ of $R$.

(b) If $R$ is commutative, $N K_{i}(R) \rightarrow \Pi N K_{i}\left(R_{\mathscr{T}}\right)$ is an injection, the product being over the maximal ideals $\mathfrak{N}$ of $R$.

Proof. By Vorst's Theorem, the result is true for $i=0,1$; for $i<0$ we reduce the proof to the case $i=0$ using the fact [1] that $N K_{i}(R)$ is a canonically defined direct summand of $N K_{0}(A), A=R\left[t_{1}, t_{1}^{-1}, \ldots, t_{-i}, t_{-i}^{-1}\right]$. For (b) we merely note that the diagram

$$
\begin{aligned}
& N K_{0}(A) \rightarrow \prod_{\max (A)} N K_{0}\left(A_{\mathscr{T} \cap R}\right) \rightarrow \prod_{\max (A)} N K_{0}\left(A_{\mathscr{T}}\right) \\
& \text { UI UI } \\
& N K_{i}(R) \quad \rightarrow \quad \prod_{\max (R)} N K_{i}\left(R_{\Im R}\right)
\end{aligned}
$$

commutes, the horizontal composition being an injection. 
For (a), given an element $\xi \in N K_{i}\left(R_{S}\right) \subseteq N K_{0}\left(A_{S}\right) \subseteq K_{0}\left(A_{S}[t]\right)$, we represent it as $[P]-r$ for some projective $A_{S}[t]$-module $P$. Let $e(t) \in M_{m}\left(A_{S}[t]\right)$ be an idempotent matrix with $P=$ image $(e)$. Now $\xi(0)=0$ in $K_{0}\left(A_{S}\right)$ so (by adding free summands to $P$ and changing basis in $\left.A_{s}^{m}[t]\right)$ we can arrange that

$$
e(0)=\left(\begin{array}{ll}
I & 0 \\
0 & 0
\end{array}\right)
$$

Hence we can find $s \in S$ so that $e(s t)$ has coefficients in $A_{s}$, and even so that $e^{2}(s t)=e(s t)$. Let $Q=$ image $(e(s t))$. As $Q(0)=$ image $e(0)$ is free, $[Q]-r$ lies in $N K_{0}(A)$. From the commutativity of

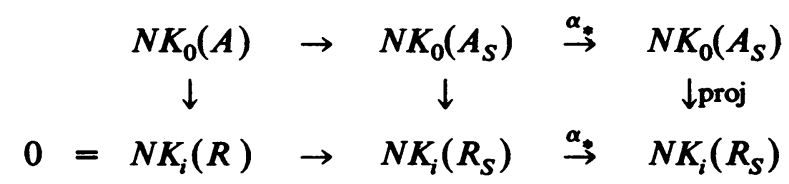

(where $\alpha$ denotes the automorphism $t \mapsto t / s$ of $R_{S}[t]$ ) we see that in $N K_{i}\left(R_{S}\right)$ we have $0=\operatorname{proj}\left(\alpha_{*}\left[Q_{s}\right]-r\right)=\xi$, since $\alpha\left(Q_{s}\right)$ is the image of $\alpha e(s t)=e(t)$, i.e., $P$. Done.

COROLlaRY 4.2. Let $R, i$ be as above. If $R$ is $K_{i}$-regular, so is $R_{s}$ for every central m.c.s. $S \subset R$. Conversely, if $R_{\Re}$ is $K_{i}$-regular for each $\mathfrak{N} \in \operatorname{Max}(R)$, $R$ commutative, then $R$ is also $K_{i}$-regular.

Proof. Let $X=\left\{x_{1}, \ldots, x_{n}\right\}$. If $R$ (hence $R[X]$ ) is $K_{i}$-regular, then $0=N K_{i}\left(R[X]_{s}\right)=N K_{i}\left(R_{s}[X]\right)$ by (a). This implies the first statement. For the converse, given $\mathscr{N} \in \operatorname{Max}(R[X])$, let $\Re$ be a maximal ideal of $R$ containing $\Re \cap R$, and note that $R[X]_{\mathscr{X}}$ is a localization of $R_{\mathscr{X}}[S]$. If $R_{\mathscr{X}}$ is $K_{i}$-regular, the above implies that $R_{\mathscr{\vartheta}}[X]_{S}=R[X]_{\mathscr{N}}$ is also $K_{i}$-regular. Varying $\mathfrak{R}$ and applying (b) of the theorem yields the $K_{i}$-regularity of $R$.

Along the same lines, we have

Proposition 4.3. Let $R$ be a commutative ring, $S$ an m.c.s. in $R$;

(a) $N \operatorname{Pic}(R)=0$ implies $N \operatorname{Pic}\left(R_{S}\right)=0$ and $N S K_{0}(R)=0$ implies $N S K_{0}\left(R_{S}\right)=0$.

(b) The maps $N \operatorname{Pic}(R) \rightarrow \Pi N \operatorname{Pic}\left(R_{\Im \mathbb{R}}\right)$ and $N S K_{0}(R) \rightarrow \Pi N S K_{0}\left(R_{\mathscr{T}}\right)$ are injections.

(c) If $R$ is Pic-regular (resp. $S K_{0}$-regular), so is $R_{S}$. Conversely, if $R_{\mathscr{T}_{R}}$ is Pic-regular (resp. $S K_{0}$-regular) for each $\mathfrak{N} \in \operatorname{Max}(R)$, so is $R$.

Proof. The proof that (a), (b) imply (c) is formally as in Corollary 4.2. Vorst's Theorem implies (b), since Pic and $S K_{0}$ are canonical subsets of $K_{0}$. For (a) we argue as follows. Given $\operatorname{det}(\xi) \in N \operatorname{Pic}\left(R_{S}\right)$, choose $\xi \in N K_{0}(R)$, $P, e, s, \alpha$, and $[Q]-r \in N K_{0}(R)$ as in the proof of Theorem 4.1. We then have

$$
1=\alpha_{*} \operatorname{det}([Q]-r)_{S}=\operatorname{det} \alpha_{*}\left(\left[Q_{S}\right]-r\right)=\operatorname{det}(\xi) .
$$

Given $\xi \in N S K_{0}\left(R_{S}\right) \subset N K_{0}\left(R_{S}\right)$, again choose $P, e, s, \alpha$, and $Q$. Then $\eta=[Q]+1-\operatorname{det}(Q)-r$ lies in $N S K_{0}(R)=0$ and $\alpha_{*} \operatorname{det}\left(Q_{S}\right)=\operatorname{det}(\xi)=1$ shows that $\alpha_{*}\left(\eta_{s}\right)=\xi$. Done. 
REMARK. Traverso [18] demonstrated the validity of this proposition for $\operatorname{Pic}(R)$ under the additional assumptions that $R$ was noetherian with finite normalization (seminormal).

Bass asked in [2] if $K_{1}$-regularity implies $K_{0}$-regularity. The affirmative answer is a special case of the next corollary.

Corollary 4.4. Let $R$ be an associative ring, $-\infty<i \leqslant 1$.

(a) $R$ is $K_{i}$-regular if and only if $R$ is Laurent $K_{i}$-regular.

(b) If $R$ is $K_{i}$-regular and $j \leqslant i, R$ is $K_{j}$-regular also.

Proof. By (4.2) if $R$ (hence $R[X]$ ) is $K_{i}$-regular, so is the localization $R\left[x_{1}, x_{1}^{-1}, \ldots, x_{n}, x_{n}^{-1}\right]$. This proves (a). Now $K_{j}$ is a contracted functor of $K_{i}$, so $N^{n} K_{j}(R)$ is a summand of $N^{n} K_{i} R\left[x_{1}, x_{1}^{-1}, \ldots, x_{m}, x_{m}^{-1}\right]$ for $m>i-j$. Since $R$ is $K_{i}$-regular iff $N^{n} K_{i}(R)=0$ for all $n \geqslant 1$, part (a) implies part (b).

REMARK. We have actually shown that $N K_{i}(R[t])=0$ implies $N K_{i-1}(R)=$ $N K_{i}\left(R\left[t, t^{-1}\right]\right)=0$.

Note that part (a) corrects Bass' example 2.2(3) of [2]. The reason is that $A$ can be Laurent $K_{0}$-regular and yet $K_{-1}(A) \neq 0$. If $A=\mathrm{Z}[\pi], \pi$ cyclic of square-free order, then $A$ is (Laurent) $K_{0}$-regular. However, $K_{i}(A)=0$ for all $i<0$ iff the order of $\pi$ is prime.

Note. Vorst and Van der Kallen [21] have independently proved (4.1), (4.2) and (4.4) for $K_{i}, 1 \leqslant i<\infty$ (assuming $R$ is commutative). Consequently, they hold for all $i \in \mathbf{Z}$ when $R$ is commutative.

In this paper, we will only need these results for $i<1$, since we are dealing with $K V$-theory. The relevant consequence of (4.4) is that $R$ is $K V_{i}$-regular for all $i \in \mathbf{Z}$ just in case $R$ is $K_{0}$-regular. If $R$ is $K_{1}$-regular, $R$ also has the property that any epi $S \rightarrow R$ is a Gl-fibration. Thus 4.4 says that $K_{1}$-regular rings are the "nice" rings for the Karoubi-Villamayor theory.

Returning to hyperplanes, we shall assume that $k$ is commutative and $K_{1}$-regular for the rest of the paper.

Proposition 4.5. If $k$ is $K_{1}$-regular and $\mathscr{F} \subset \operatorname{LIN}(A)$ is admissible, then $k[\mathcal{C}(\mathcal{F})]$ is $K_{i}$-regular, $i<1$.

Proof. Set $\Pi X=x_{0} \cdots x_{n}$. By [4] the ring $k\left[x_{0}, \ldots, x_{n}\right] /(\Pi X)=$ $k[\mathcal{C}(X)]$ is $K_{1}$-regular if $k$ is. We now use the criterion of (4.2) for $R=$ $k[\mathcal{C}(\mathscr{F})]$. Given $\mathfrak{N} \in \operatorname{Max}(R)$, note that $\sigma=\mathscr{F} \cap \mathfrak{N}$ is an element of $\mathcal{C}(\mathscr{F})$ or empty. By the remarks after the definition of admissible set, we can in fact assume $\sigma \subset X \subset \mathscr{F}$. By Theorem $1.11, R_{\Re}=(A / F A)_{\mathscr{T}}$ and this is a localization of $A /(\Pi X)$. Hence $R_{\mathscr{T}}$ is $K_{1}$-regular for each $\Re$, and hence $R$ is $K_{1}$-regular.

From [8] and (1.11) we have

CoRollary 4.6. If $\mathscr{F} \subset \operatorname{LIN}(A)$ is admissible, $A \rightarrow k[\mathcal{C}(\mathcal{F})]$ is a Gl-fibration.

For a $k$-algebra $R$ we define $\tilde{K} V_{i}(R)$ to be the cokernel of $K V_{i}(k) \rightarrow$ $K V_{i}(R)$. If $I$ is an ideal of $R$ for which $R / I \cong k$ as $k$-algebras, we call $I$ 
$k$-rational; we can identify $\tilde{K} V_{i}(R)$ with the resulting kernel of $K V_{i}(R) \rightarrow$ $K V(k)$. This subgroup in general depends on $I$. For example, if $\mathbb{Q}$ is a $k[x]$-poset, $k[\mathbb{Q}]=k \oplus \cdots \oplus k$ and each $K V_{i}(k[\mathbb{Q}]) \rightarrow K V_{i}(k[x] /(f)), f \in$ $Q$, is projection onto a component.

However, the argument of $[14$, p. 519] shows that $(i \in \mathbf{Z})$.

LEMMA 4.7. If $|\mathfrak{Q}|$ is connected, the map $K V_{i}(k[\mathbb{Q}]) \rightarrow K V_{i}(k)$ is independent of the choice of $k$-rational $I \subset k[Q]$. Hence there is a canonical decomposition $K V_{i}(k[\mathbb{Q}])=K V_{i}(k) \oplus \tilde{K} V_{i}(k[\mathbb{Q}])$.

We note that if $n \neq 0, \mathscr{F}$ admissible, then $|\mathcal{C}(\mathscr{F})|$ is connected: each hyperplane meets one element of any fixed coordinate system.

Here is another consequence of Proposition 4.5.

Corollary 4.8. Let $\mathcal{F} \subset \operatorname{LIN}(A)$ be admissible, and suppose every coordinate system of $\mathscr{F}$ contains some fixed $f_{0} \in \mathcal{F}$. Then $\tilde{K} V_{i}(k[\mathcal{C}(\mathscr{F})])=0$.

Proof. A change of coordinates allows us to assume $X \subset \mathscr{F}$ and $x_{0}=f_{0}$. Let $\iota: A / x_{0} A \rightarrow A$ be the inclusion $\iota\left(\bar{x}_{i}\right)=x_{i}$. Now every $f \in \mathscr{F}-X$ must lie in $\iota\left(A / x_{0} A\right)$ : otherwise $\{f\} \cup X-\left\{x_{0}\right\}$ would be a coordinate system in $\mathscr{F}$.

Define $\tilde{\alpha}: A \rightarrow A[t]$ by $\tilde{\alpha}\left(x_{0}\right)=t x_{0}, \tilde{\alpha}\left(x_{i}\right)=x_{i}$ otherwise. Then $\tilde{\alpha}(f)=f$ for all $f \in \mathscr{F}-\left\{x_{0}\right\}$, so by Theorem 1.11 a map $\alpha: R \rightarrow R[t]$ is induced, $R=k[\mathcal{C}(\mathscr{F})]$. The diagram

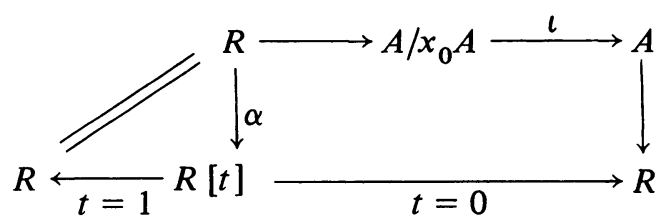

commutes. Apply $\tilde{K} V_{i}$ and note that by (4.5) the maps $\tilde{K} V_{i}(t=0), \tilde{K} V_{i}(t=1)$ are isomorphisms. Hence $\alpha_{*}$ is an isomorphism factoring through $\tilde{K} V_{i}(A)=$ 0. This shows $\tilde{K} V_{i}(R)=0$, as desired.

5. $K$-theory. We assume that $k$ is $K_{1}$-regular for this section.

Proposition 5.1. If $\delta$ is a polysimplicial $n$-sphere, $\tilde{K} V_{i}(k[\delta])=K V_{n+i}(k)$ for all $i \in \mathbf{Z}$.

Proof. If $n=0, k[\mathcal{\delta}] \simeq k \oplus k$ and the result is clear. Inductively, let $\mathcal{S}=\mathcal{S}\left(n_{1}, \ldots, n_{k}\right)=\mathcal{C}(\mathscr{F})$ and write $A=k\left[x_{10}, \ldots, x_{k n_{k}}\right]$ as in Definition 2.1. By the example after (1.7),

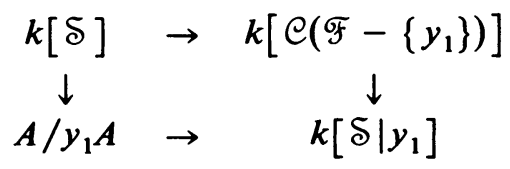

is a cartesian square. Now every coordinate system in $\mathscr{F}-\left\{y_{1}\right\}$ includes $x_{10}$, so by Corollary 4.8 we have $\tilde{K} V_{*}\left(k\left[\mathcal{C}\left(\mathcal{F}-\left\{y_{1}\right\}\right)\right]\right)=0$. We also have $\tilde{K} V_{*}\left(A / y_{1} A\right)=0$. By Lemma $2.2, \delta \mid y_{1}$ is a polysimplicial $(n-1)$-sphere, so 
by Corollary 4.6 the bottom arrow is a Gl-fibration. From induction and the resulting long exact sequence we obtain for all $i \in \mathbf{Z}$

$$
\tilde{K} V_{i}(k[\delta]) \simeq \tilde{K} V_{i+1}\left(k\left[\delta \mid y_{1}\right]\right) \simeq K V_{n+i}(k) \text {. }
$$

REMARK. Earlier versions of this computation were [15] for $n=1$, [16] for $n=2,[4]$ for $\delta(n)$ and $[3]$ for $\delta(0, \ldots, 0)$.

The following theorem is to be regarded as a $K$-theoretic analogue of the topological result (3.13).

THEOREM 5.2. If $\Sigma$ is a basic set of $n$-spheres for the admissible set $\mathscr{F}$, the morphisms $\left(\lambda_{i}, \varphi_{i}\right): \mathcal{S}_{i} \rightarrow \mathcal{C}(\mathscr{F})$ induce an isomorphism

$$
\tilde{K} V_{*}(k[\mathcal{C}(\mathscr{F})]) \rightarrow \prod_{\Sigma} \tilde{K} V_{*}\left(k\left[\delta_{i}\right]\right) \text {. }
$$

Proof. Since $k[\delta(0)]=k \oplus k$, the result for $n=0$ follows from the definition of basic 0 -sphere. If $\mathscr{F}=X$ the result follows from Corollary 4.8. Now assume card(F) $>n+1$. Let $f, \Sigma_{0}$ be as in Definition 2.6. By the example after (1.7) we have the cartesian square

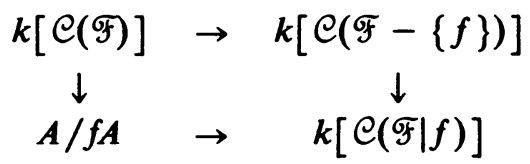

the bottom map being a Gl-fibration by (4.6). For each $\left(\lambda_{i}, \varphi_{i}\right) \in \Sigma_{0}$ we obtain a map of cartesian squares, hence a commutative diagram:

$$
\begin{gathered}
\ldots \rightarrow \tilde{K} V_{*+1}(k[\mathcal{C} \mid f]) \stackrel{\partial}{\rightarrow} \tilde{K} V_{*}(k[\mathcal{C}(\mathscr{F})]) \rightarrow \tilde{K} V_{*}(k[\mathcal{C}(\mathscr{F}-\{f\})]) \rightarrow \ldots \\
\downarrow \\
\coprod_{\Sigma_{0}} \tilde{K} V_{*+1}\left(k\left[\delta_{i} \mid s_{i}\right]\right) \rightarrow \coprod_{\Sigma_{0}} \tilde{K} V_{*}\left(k\left[\delta_{i}\right]\right)
\end{gathered}
$$

Inductively, the left vertical arrow is an isomorphism. By Proposition 5.1 the bottom arrow is an isomorphism, so the right vertical arrow is a split epi and $\partial$ is a split monic. Hence the top sequence splits. The result follows, since by induction the remaining term is

$$
\coprod_{\Sigma-\Sigma_{0}} \tilde{K} V_{*}\left(k\left[\delta_{i}\right]\right)
$$

Since $\mathcal{F}$ always has a basic set $\Sigma$ by (2.8) and $\operatorname{card}(\Sigma)=g(\mathcal{C}(\mathcal{F}))$ by (3.13), Theorems 1.11, 4.5, and 5.2 yield (a) and (b) of

Corollary 5.3. Let $\mathcal{F}$ be an admissible set for $A=k\left[x_{0}, \ldots, x_{n}\right]$. Set $F=\Pi \mathscr{F}, g=g(\mathcal{C}(\mathscr{F}))$, and assume $k$ is $K_{1}$-regular. Then

(a) $K V_{i}(A / F A)=K V_{i}(k) \oplus g K V_{n+i}(k), i \in \mathbf{Z}$.

(b) $K_{i}(A / F A)=K_{i}(k) \oplus g K_{i}(k),-\infty<i<1$.

(c) If $n=1$,

$$
\begin{aligned}
S K_{0}(A / F A) & =S K_{0}(k) \oplus g S K_{1}(k), \\
S K_{1}(A / F A) & =S K_{1}(k) \oplus g K V_{2}(k), \\
\operatorname{Pic}(A / F A) & =\operatorname{Pic}(k) \oplus g U(k), \\
U(A / F A) & =U(k) .
\end{aligned}
$$


(d) If $n>2$,

$$
\begin{aligned}
S K_{0}(A / F A) & =S K_{0}(k) \oplus g K V_{n}(k), \\
S K_{1}(A / F A) & =S K_{1}(k) \oplus g K V_{n+1}(k), \\
\operatorname{Pic}(A / F A) & =\operatorname{Pic}(k), \\
U(A / F A) & =U(k) .
\end{aligned}
$$

The formulas for (c) and (d) follow from replacing the long exact $K V$ sequence with the $S K V$ and Picard sequences [1], [22] in Proposition 5.1, and using the naturality in Theorem 5.2.

From the fact that $K_{*}(k)=K V_{*}(k)$ and is 0 for $*<0$ when $k$ is a regular ring we obtain (using Proposition 3.5)

COROllaRY 5.4. Let $k$ be a commutative regular ring. Then with $n, \mathcal{F}, g, F$ as in (5.3) we have

(a) $K V_{i}(A / F A)=K_{i}(k) \oplus g K_{n+i}(k), i \in \mathbf{Z}$.

(b) $K_{i}(A / F A)=K_{i}(k) \oplus g K_{n+i}(k), i=0,1$.

(c) $K_{-i}(A / F A)=g K_{n-i}(k), 1<i \leqslant n$.

(d) $K_{-i}(A / F A)=0, i>n$.

If the $N$ hyperplanes of $\mathcal{F}$ are in general position, the number $g$ is $\left(\begin{array}{l}N-1 \\ n+1\end{array}\right)$.

We conclude this section with a description of the multiplicative structure of $K V_{*}(k[\mathcal{C}]), \mathcal{C}=\mathcal{C}(\mathscr{F})$, defined by Karoubi [10]. Recall that $K V_{*}(k)$ is a $\mathrm{Z}$-graded ring. We will consider the integral cohomology ring $\boldsymbol{H}^{-*}(|\mathcal{C}|)$ as graded in negative dimensions to make degrees add properly.

Assertion 5.5. There is an isomorphism of graded rings

$$
K V_{*}(k[\mathcal{C}]) \cong K V_{*}(k) \otimes_{\mathrm{z}} H^{-*}(|\mathcal{C}|)
$$

Sketch of Proof. From Proposition 3.13 and Karoubi's axiom (2a) for multiplication [10, p. 78], the identification in Theorem 5.2 yields an isomorphism of graded $K V_{*}(k)$-modules. There is no problem when $n=0$, so assume $n>0$. Since multiplication in $\tilde{H}^{*}\left(||_{\mid}\right)$is trivial, it is enough to show that multiplication in the ideal $\tilde{K} V_{*}(k[\mathcal{C}])$ is trivial. This can be accomplished by using the cartesian squares in the proof of 5.2 and Karoubi's axiom (2a).

6. $K_{2}$ of hyperplanes. In this section we compute $K_{2}$ and $\left[K_{3}\right]$ of $k[\mathcal{C}(\mathscr{F})]$ under the assumption that the ground ring $k$ is $K_{1}$ - and $K_{2}$-regular. Here $\mathscr{F}=\left\{f_{0}, \ldots, f_{N}\right\}$ is an admissible set for $A=k\left[x_{0}, \ldots, x_{n}\right]$. Set $F_{i}=$ $f_{0} \ldots \hat{f}_{i} \ldots f_{N}$ and $F=f_{i} F_{i}=f_{0} \ldots f_{N}$, and let $R$ denote $k[\mathcal{C}(\mathscr{F})]=A / F A$. We will write $\bar{R}$ for $A / f_{0} A \oplus \cdots \oplus A / f_{N} A$, recognizing the slight notational abuse when $k$ is not integrally closed (see Proposition 1.12).

As in [5] we shall write $D \tilde{K}_{2}(R)$ for the subgroup of $\tilde{K}_{2}(R)$ generated by all $\langle a, b\rangle$ with $a b=0$. For information on these symbols, we refer the reader to [6], [7], [19]. In this case we have relations $\langle a, b+c\rangle=\langle a, b\rangle+\langle a, c\rangle$ and $\langle a, b r\rangle=\langle a r, b\rangle$ for $a b=0, r \in R$. Contrary to the usual convention we write $D \tilde{K}_{2}(R)$ additively. As in (4.7) we can assume $D \tilde{K}_{2}(R) \subset K_{2}(R)$. 
LEMMA 6.1. (i) $\left\langle a, b^{2}\right\rangle=0$ if $a b=0$.

(ii) $D \tilde{K}_{2}(R)$ is in the kernel of $\tilde{K}_{2}(R) \rightarrow \tilde{K} V_{2}(R)$.

(iii) $\Sigma\left\langle f_{i}, F_{i} h_{i}\right\rangle=0$ for $h \in R$.

Proof. Relation (i) is immediate. The element $\langle a, b t\rangle \in \tilde{K}_{2}(R[t])$ is a contraction of $\langle a, b\rangle$, showing that $\langle a, b\rangle$ maps to zero in any homotopy functor, hence (ii). For (iii) we show that if $a_{0} \ldots a_{N}=0$ in any ring $R$ then $\Sigma\left\langle a_{i}, a_{0} \ldots \hat{a}_{i} \ldots a_{N}\right\rangle=0$. This is clear for $N=1$ and induction on $\left(a_{0} a_{1}\right) a_{2} \ldots a_{N}$ yields the result (iii). The key relation here is $\langle a b, c\rangle=$ $\langle a, b c\rangle+\langle b, a c\rangle$ if $a b c=0$.

THEOREM 6.2. If $k$ is $K_{1}$ - and $K_{2}$-regular the sequence

$$
0 \rightarrow D \tilde{K}_{2}(R) \rightarrow K_{2}(R) \rightarrow K V_{2}(R) \rightarrow 0
$$

is exact, and $D \tilde{K}_{2}(R)$ is generated as an $R$-module by the $N+1$ elements $\left\langle f_{i}, F_{i}\right\rangle$.

Proof. This is proven in exactly the same fashion as Proposition 3.5 of [5], so we sketch the main points. Consider the map $\varphi: k\left[t_{0}, \ldots, t_{N}\right] \rightarrow A$ given by $\varphi\left(t_{i}\right)=f_{i}$. Set $I=\operatorname{Ker}(\varphi), J=\left(\Pi t_{i}\right)$ and $S=k\left[t_{0}, \ldots, t_{N}\right] / I J$. Now $I \cap$ $J=I J$ : if $g \Pi t_{i} \in I$ then $\varphi(g) F=0$ in $A$, hence $\varphi(g)=0$ since $F$ is a nonzero divisor. This yields the cartesian square

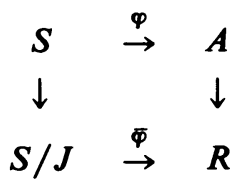

Comparing Mayer-Vietoris sequences, we obtain

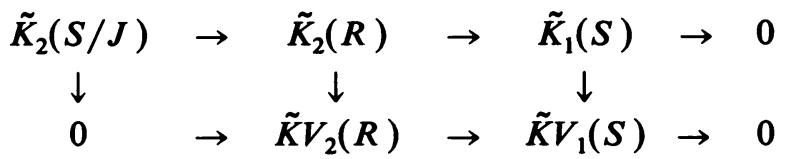

We claim that $S$ is $K_{1}$-regular. From this it follows that

$$
\tilde{K}_{2}(S / J) \rightarrow \tilde{K}_{2}(R) \rightarrow \tilde{K} V_{2}(R) \rightarrow 0
$$

is exact. By [7] $\tilde{K}_{2}(S / J)$ is $D \tilde{K}_{2}(S / J)$ and is generated by the $N+1$ symbols $\left\langle t_{i}, t_{0} \ldots \hat{t}_{i} \ldots t_{N}\right\rangle$. Apply $\bar{\varphi}$ to $\tilde{K}_{2}(S / J)$ and add $K_{2}(k)$ to $\tilde{K}_{2}(R)$ and $\tilde{K} V_{2}(R)$. The theorem follows from Lemma 6.1(ii).

It remains to show that $S$ is $K_{1}$-regular. By Proposition 4.1 it is enough to check $S$ locally. We have already noticed that $A$ and $S / J$ are $K_{1}$-regular if $k$ is. If $I \not \subset \mathfrak{p}, S_{\mathfrak{p}}=(S / J)_{\mathfrak{p}}$; if $J \not \subset \mathfrak{p}$ we have $S_{\mathfrak{p}}=A_{\mathfrak{p}}$. By (4.1) these are $K_{1}$-regular. We are reduced to the case $I+J \subset \mathfrak{p}$. The admissibility of $\mathscr{F}$ implies that $\mathscr{F} \cap \varphi(\mathfrak{p})$ is contained in some coordinate system of $A$; we can assume this coordinate system to be $\left\{x_{0}, \ldots, x_{n}\right\}$. Let $T=$ $k\left[t_{0}, \ldots, t_{N}\right] /\left(t_{0} \ldots t_{n}\right) I$, so that $S_{\mathfrak{p}}=T_{p}$. In the cartesian square

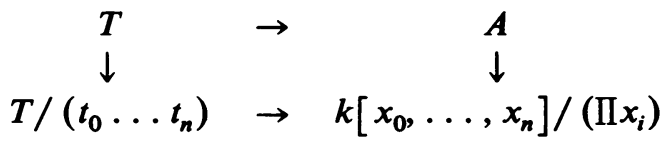


the bottom arrow splits by $x_{i} \mapsto t_{i}$. By [4] this implies that $T$, hence $T_{\mathfrak{p}}$ is $K_{1}$-regular. Done.

We now define a map $[-]: \bar{R} \rightarrow D \tilde{K}_{2}(R)$. Given $h_{0}, \ldots, h_{N} \in A$ we set $\left[\left(\bar{h}_{0}, \ldots, \bar{h}_{N}\right)\right]=\Sigma\left\langle f_{i}, F_{i}\right\rangle h_{i}$. By (6.1) (i) and (iii) we see that $\left[\left(\bar{h}_{0}, \ldots, \bar{h}_{N}\right)\right]$ is a well-defined group map and that the composite [diag]: $A \rightarrow \bar{R} \rightarrow D \tilde{K}_{2}(R)$ is zero. By Theorem 1.11 the image $\operatorname{diag}(A) \subset \bar{R}$ is $R$. Hence:

LEMMA 6.3. There is a well-defined group homomorphism [-]: $\bar{R} / R \rightarrow$ $D \tilde{K}_{2}(R)$ taking $\left(0, \ldots, h_{i}, 0, \ldots, 0\right)$ to $\left\langle f_{i}, F_{i} h_{i}\right\rangle$.

From Theorem 6.2 we see that $[-]$ is onto. We shall show that it is an isomorphism. To this end we recall some facts about the modified Tate map. Gersten [9] defined a map dlog: $K_{2}(R) \rightarrow \Omega_{R}^{2}$; the Tate map is - dlog (see [6], noting the sign error). R. K. Dennis has shown [23] that $-\operatorname{dlog}\{u, v\}=$ $(u v)^{-1} d u \wedge d v$ implies that $-\operatorname{dlog}(\langle a, b\rangle)=(1+a b)^{-1} d a \wedge d b$ for all pointy brackets $\langle a, b\rangle$.

Let $\mathrm{c}$ denote the conductor $\left(F_{0}, \ldots, F_{N}\right)$ from $\bar{R}$ to $R$, as in Proposition 1.12. A straightforward computation shows that $\Omega_{R / k} \otimes R / \mathrm{c} \cong(R / \mathrm{c})^{n+1}$ on basis $\left\{d x_{i}\right\}$. We let $D: R \rightarrow \Omega_{R / k} \otimes R / \mathrm{c}$ be the natural map, and note that we have the formula $D h=\Sigma\left(\partial h / \partial x_{i}\right) d x_{i}$. We shall refer to the composite

$$
D \tilde{K}_{2}(R) \subset K_{2}(R) \rightarrow \Omega_{R}^{2} \rightarrow \Omega_{R / k}^{2} \otimes R / \mathfrak{c} \approx \Lambda^{2}(R / \mathrm{c})^{n+1}
$$

as "Tate".

LEMMA 6.4. Write $F_{i j}=F / f_{i} f_{j}=f_{0} \ldots \hat{f}_{i} \ldots \hat{f}_{j} \ldots f_{N}$. Then $\operatorname{Tate}\left(\left\langle f_{i}, F_{i} h\right\rangle\right)$ $=h D f_{i} \wedge \Sigma_{j} F_{i j} D f_{j}$. The terms $D f_{i} \wedge D f_{j}(i \neq j)$ are unimodular unless $\left(f_{i}, f_{j}\right) A$ $=A$, in which case $D f_{i} \wedge D f_{j}=0$.

Proof. $\left\langle f_{i}, F_{i} h\right\rangle$ goes to $d f_{i} \wedge d F_{i} h=d f_{i} \wedge\left(F_{i} d h+h d F_{i}\right)$ in $\Omega_{R / k}^{2}$. Since $F_{i} \in \mathrm{c}$ this goes to $h\left(D f_{i} \wedge D F_{i}\right)$ in $\Omega_{R / k}^{2} \otimes R / \mathrm{c}$ whence the first statement. If $\left(f_{i}, f_{j}\right) A=A$ then $f_{j}=a f_{i}-b$ for $a, b \in k$, so $D f_{i} \wedge D f_{j}=0$. Otherwise we can change coordinates in $A$ to make $f_{i}=x_{0}, f_{j}=x_{1}$, in which case it is clear that $d x_{0} \wedge d x_{1}$ is unimodular.

Now $\bar{R} / R$ is an $R / \mathrm{c}$-module and the composite Tate $([-]): \bar{R} / R \rightarrow$ $\Lambda^{2}(R / \mathrm{c})^{n+1}$ is an $R / \mathrm{c}$-module homomorphism.

Proposition 6.5. The map Tate $([-])$ is an injection. Hence the map [-]: $\bar{R} / R \rightarrow D \tilde{K}_{2}(R)$ is an isomorphism.

Proof. The second statement follows from the first, given Theorem 6.2. Let $h_{i} \in A$ and suppose that $0=\operatorname{Tate}\left[\left(\bar{h}_{0}, \ldots, \bar{h}_{N}\right)\right]=\sum_{i} h_{i} D f_{i} \wedge \Sigma_{j} F_{i j} D f_{j}$. We will show that $\left(\bar{h}_{0}, \ldots, \bar{h}_{N}\right) \in R$ by the criterion (1.10). For each $p, q$ tensor with the quotient $A /\left(f_{p}, f_{q}\right)$ of $R / c$ to get the equation

$$
\left(h_{p} F_{p q}-h_{q} F_{q p}\right) D f_{p} \wedge D f_{q} \equiv 0 \bmod \left(f_{p}, f_{q}\right) .
$$

If $\left(f_{p}, f_{q}\right) A=A$ there is nothing to show, so we assume otherwise. Since $F_{q p}=F_{p q}$ is a nonzero divisor and $D f_{p} \wedge D f_{q}$ is unimodular, we obtain $h_{p} \equiv h_{q} \bmod \left(f_{p}, f_{q}\right)$. Done. 
Corollary 6.6. Let $k$ be $K_{1}$ - and $K_{2}$-regular, $A=k\left[x_{0}, \ldots, x_{n}\right]$, and let $\mathscr{F}=\left\{f_{0}, \ldots, f_{N}\right\}$ be an admissible set of linear polynomials in $A$. Set $R=$ $A /\left(\Pi f_{i}\right), \bar{R}=\Pi A /\left(f_{i}\right)$. Then there is a short exact sequence

$$
0 \rightarrow \bar{R} / R^{[-]} \rightarrow K_{2}(R) \rightarrow K V_{2}(R) \rightarrow 0 .
$$

If $k$ is integrally closed, $\bar{R}$ is the normalization of $R$.

REMARK. As this sequence is natural in $k$, we see that $N K_{2}(R) \simeq(\bar{R} / R) \otimes$ $k[t]$. This shows that $R$ is not $K_{2}$-regular (unless $n=0$ ).

REMARK. In general we see no way to split the sequence of (6.6). If $k$ is a field, though, the injection Tate([-]) splits as a vector space map over $k$, and the map $K_{2}(R) \rightarrow \Lambda^{2}(R / \mathrm{c})^{n+1} \rightarrow \bar{R} / R$ splits the injection [-]. In fact this argument only requires $k$ to contain a field.

Here are other cases in which (6.6) splits. If $g=0$ then $K V_{2}(R)=K_{2}(k)$ and the splitting is structural. If $n=0, \bar{R} / R=0$. If $n=1, \bar{R} / R=(N+1) k$ $\simeq R / \mathrm{c}=\Lambda^{2}(R / \mathrm{c})^{2}$ and Tate $([-])$ is an isomorphism; the splitting in this case was obtained in a different way in [5]. Splitting in the cases $N=n$ and $N=n+1$ was obtained in [7], [4]; the complexity of their computations illustrates the problems in obtaining a splitting in general.

We summarize this discussion:

Corollary 6.7. Let $k, A, \mathcal{F}$ be as in (6.6), and set $g=g(\mathcal{C}(\mathcal{F}))$. If $g=0$, $n=0$ or $1, \operatorname{card}(\mathscr{F})=n+1$ or $n+2$, or if $k$ contains a field then

$$
K_{2}(R) \simeq K_{2}(k) \oplus g K V_{n+2}(k) \oplus \bar{R} / R \text {. }
$$

REMARK. If $n=1, \bar{R} / R$ is a free $k$-module of rank $m_{0}$. If $n>2, \bar{R} / R$ can be shown to be a free $k$-module of countably infinite rank. This leads to the noncanonical identifications of $D \tilde{K}_{2}(R)$ in [7].

Finally, we use the methods of [22] to obtain information on $K_{3}$.

TheOREM 6.8. Let $k$ be $K_{1}$ - and $K_{2}$-regular, $\mathcal{F}$ an admissible set and $R=A /(\Pi \mathscr{F})=k[\mathcal{C}(\mathscr{F})]$. Then

$$
N K_{3}(R) \rightarrow K_{3}(R) \rightarrow K V_{3}(R) \rightarrow 0
$$

is an exact sequence. Here $N K_{3}(R)$ is the kernel of " $t=0$ ": $K_{3} R[t] \rightarrow K_{3} R$ and is mapped to $K_{3}(R)$ by " $t=1$ ".

Proof. Consider the Gersten-Anderson spectral sequence $(p>0, q>1)$

$$
E_{p q}^{1}=K_{q}\left(R\left[t_{1}, \ldots, t_{p}\right]\right) \Rightarrow K V_{p+q}(R) \text {. }
$$

Since $R$ is $K_{1}$-regular we have $E_{p 1}^{2}=0$ for $p \neq 0$. We also have $E_{0 q}^{2}=\left[K_{q}\right] R$ for $q>1$, where $\left[K_{q}\right] R$ is the cokernel of $N K_{q}(R) \rightarrow K_{q}(R)$. We will be done if we show that $E_{p 2}^{2}=0$ for $p \neq 0$.

There is a fibration of simplicial groups

$$
0 \rightarrow D \tilde{K}_{2}\left(R_{*}\right) \rightarrow K_{2}\left(R_{*}\right) \rightarrow K V_{2}\left(R_{*}\right) \rightarrow 0 .
$$

The right-hand complex is constant and the left-hand complex is just $(\bar{R} / R)$ $\otimes k_{*}$. This is acyclic, i.e., $\pi_{p}\left(D \tilde{K}_{2}\left(R_{*}\right)\right)=0$ for all $p$. The long exact homotopy sequence gives us $E_{p 2}^{2}=\pi_{p}\left(K V_{2}\left(R_{*}\right)\right)$, which is 0 for $p \neq 0$. 


\section{REFERENCES}

1. H. Bass, Algebraic K-theory, Benjamin, New York, 1968.

2. Some problems in "classical" algebraic K-theory, Lecture Notes in Math., vol. 342, Springer-Verlag, New York, 1973.

3. B. Dayton, Karoubi-Villamayor $K$-theory of cubical rings (preprint).

4. $\ldots$ K-theory of tetrahedra, J. Algebra 56 (1979), 129-144.

5. B. Dayton and L. Roberts, $K_{2}$ of $\boldsymbol{n}$ lines in the plane, J. Pure Appl. Algebra (to appear).

6. K. Dennis and M. Stein, The functor $K_{2}:$ a survey of computations and problems, Lecture Notes in Math., vol. 342, Springer-Verlag, New York, 1973.

7. K. Dennis and M.,"Krusemeyer, $K_{2}(A[X, Y] / X Y)$, a problem of Swan, and related computations, J. Pure Appl. Algebra (to appear).

8. S. Gersten, K-theory of Lourent polynomials, Proc. Amer. Math. Soc. 30 (1971), 223-228.

9. __ Some exact sequences in the higher $K$-theory of rings, Lecture Notes in Math., vol. 341, Springer-Verlag, New York, 1973.

10. M. Karoubi, La périodicité de Bott en K-théorie générale, Ann. Sci. École Norm. Sup. 4 (1971), 63-95.

11. M. Karoubi and $O$. Villamayor, $K$-théorie algébrique et $K$-théorie topologique, Math. Scand. 28 (1971), 265-307.

12. F. Orecchia, Sul gruppo di Picard di certe algebre finite non integre, Ann. Univ. Ferrara Sez. VII. (to appear).

13. D. Quillen, Higher algebraic K-theory. I, Lecture Notes in Math., vol. 341, Springer-Verlag。 New York, 1973.

14. L. Roberts, The $K$-theory of some reducible affine varieties, J. Algebra 35 (1975), 516-527.

15. ㄴ. The $K$-theory of some reducible affine curves: a combinatorial approach, Lecture Notes in Math., vol. 551, Springer-Verlag, New York, 1976.

16. _. The Picard group of planes in 3-space (preprint).

17.,$S K_{1}$ of $n$ lines in the plane, Trans. Amer. Math. Soc. 222 (1976), 353-365.

18. C. Traverso, Seminormality and Picard group, Ann. Scuola Norm. Sup. Pisa 24 (1970), 585-595.

19. W. Van der Kallen, H. Maazen and J. Stienstra, $A$ presentation for some $K_{2}(n, R)$, Bull. Amer. Math. Soc. 81 (1975), 934-935.

20. A. Vorst, Polynomial extensions and excision for $K_{1}$, Utrecht University preprint 63, 1977.

21. , Localisations of the $K$-theory of polynomial extensions, Utrecht University preprint $80,1978$.

22. C. Weibel, Nilpotence in algebraic $K$-theory, J. Algebra (to appear).

23. R. K. Dennis, Differentials in algebraic $K$-theory (unpublished).

Department of Mathematics, Northeastern Illinois University, Chicago, Illinois 60625 (Current address of B. H. Dayton)

School of Mathematics, Instrtute por Advanced Study, Princeton, New Jersey 08540

Current address (C. A. Weibel): Department of Mathematics, University of Pennsylvania, Philadelphia, Pennsylvania 19104 